\title{
Financial asset bubbles in banking networks
}

\author{
Francesca Biagini*† Andrea Mazzon* Thilo Meyer-Brandis* \\ December 20, 2018
}

\begin{abstract}
We consider a banking network represented by a system of stochastic differential equations coupled by their drift. We assume a core-periphery structure, where banks in the core hold a bubbly asset. Investments are modelled by the weight of the links, which is a function of the robustness of the banks. In this way, a preferential attachment mechanism of the banks in the periphery towards the core takes place during the growth of the bubble. We then investigate how the bubble distorts the shape of the network, both for finite and infinitely large systems, assuming a non vanishing impact of the core on the periphery. Due to the influence of the bubble, banks are no longer independent, and the strong law of large numbers cannot be directly applied to the average of banks' investments towards the periphery. This results in a term in the drift of the diffusions which does not average out, increasing systemic risk when the bubble bursts. We test this feature of the model by numerical simulations.
\end{abstract}

Keywords: Bubbles, Systemic risk, Financial networks, Mean field models

\section{Introduction}

In this paper we study the impact of financial asset bubbles on the evolution of dependence structures and systemic risk in banking networks, both for finite and infinitely large systems.

Systemic risk has been recently studied with different approaches. One stream of research aims at extending the traditional regulatory framework of monetary risk measures,

*Workgroup Financial and Insurance Mathematics, Department of Mathematics, Ludwig-Maximilians Universität, Theresienstraße 39, 80333 Munich, Germany. Emails: biagini@math.lmu.de, meyerbrandis@math.lmu.de, mazzon@math.lmu.de.

†Secondary affiliation: Department of Mathematics, University of Oslo, Box 1053, Blindern, 0316, Oslo, Norway. 
that quantify the risk of financial institutions based on a stand alone basis, to multivariate systemic risk measures that take as a primitive the whole financial system. For an overview about this topic, see Biagini et al. [8, 9], Bisias et al. [12], Chen et al. [19], Drapeau et al. [29], Feinstein et al. [34], Hoffmann et al. [43, 44], Kromer et al. [56] and references therein.

Another popular ansatz to analyse systemic risk is based on explicit network models for the financial system and the study of potential default cascades due to various contagion effects. In the seminal work of Eisenberg and Noe [31] and its many extensions (see e.g. Hurd [46] and references therein) cascade processes in static, deterministic network models are analysed by computing endogenously determined clearing/equilibrium payment vectors. Within the framework of random graph theory, cascade processes are studied in large financial random networks by means of law-of-large number effects in Amini and Minca [3, Amini et al. [4, 5], Detering et al. [26, 27, 28] and Hurd [46], and in finite random networks by Elliott et al. 32, Gai and Kapadia [38].

The approach we present in this paper is placed within the theory of mean-field equations first introduced in the influential papers of McKean [61, 62]. In recent years, this framework has been applied to the study of systemic risk in large financial networks where, contrary to the static network models mentioned above, the dynamic evolution of a network of interacting financial institutions is studied by means of a system of interacting diffusions. In this setting the diffusions represent e.g. the wealth, monetary reserves, or other more general indicators for the health of financial institutions, and are tied together through a term in the drift that implies the network structure. A first simple model in this direction is given in Fouque and Sun [37], where a system of SDEs is proposed with dynamics

$$
d X_{t}^{i}=\frac{\lambda}{n} \sum_{j=1}^{n}\left(X_{t}^{j}-X_{t}^{i}\right) d t+\sigma d W_{t}^{i}, \quad 0 \leq t<\infty
$$

where $W=\left(W_{t}^{1}, \ldots, W_{t}^{n}\right)_{t \geq 0}$ is a standard $n$-dimensional Brownian motion and $\lambda, \sigma>0$. Here, the $X^{i}$ stand for log-monetary reserves of banks, and the drift terms $\lambda\left(X_{t}^{j}-X_{t}^{i}\right)$ represent the connections between banks in the network. In this case, the borrowing and lending rate $\lambda$ is supposed to be the same for every pair of banks. When the network size $n$ grows towards infinity, it is a well-know result (see Sznitman [70]) that due to law-of-largenumber effects the diffusions in (1.1) converge towards their mean-field limit

$$
d \bar{Y}_{t}^{i}=\lambda\left(\mathbb{E}\left[\bar{Y}_{t}\right]-\bar{Y}_{t}^{i}\right) d t+\sigma d W_{t}^{i}, \quad 0 \leq t<\infty
$$

Thus, for large networks propagation of chaos applies and the evolution of the $X^{i}$ asymptotically de-couples due to averaging effects, which allows to asymptotically describe the complex system by a representative particle evolution. The model in (1.1) to study systemic risk has been generalised in various ways in a number of articles, see for example Fang et al. [33, where heterogeneity is introduced by allowing for different $\lambda_{i}$ and $\sigma_{i}$ for every bank in 
(1.1), Carmona et al. [17, 18] and Maheshwari and Sarantsev [58], where mean-field games are considered, Fouque and Ichiba [36] where the probability distributions of multiple default times is approximated, Garnier et al. [39, 40] and Battiston et al. [7] where a tradeoff between individual and systemic risk in a banking network is described, and Chong and Klüppelberg [22], Kley et al. [55] where partial mean-field limits are studied. We also mention the work of Bo and Capponi [13, where a system of jump diffusions processes is introduced with a banking sector indicator depending on positive or negative announcements, and Hambly et al. [41], where distances-to-default of financial institutions is studied in a model where herd behaviour and common exposures can lead to a structural contagion mechanism.

In this paper, the main objective is to extend the model in 1.1 in order to study the effect of a financial speculation bubble on the evolution of the network and on propagation of systemic risk. It is a common understanding that bubbles are intimately connected with financial crises, and many historical crises indeed originated after the burst of a bubble (e.g. the Great Depression of the 1930s and the financial crisis of 2007-2008). This causality is investigated for example in Brunnermeier [14] and statistically confirmed in Brunnermeier and Schnabel [16]. However, it seems that literature on mathematical models that deal with this question is very scarce.

We here specify a model for the network of financial robustness of the institutions, introduced by Battiston et al. [7] and Hull and White [45] as an indicator of agent's creditworthiness or distance to default and also considered in Kley et al. [55], by a system of coupled diffusions. In particular, we are able to include in the robustness dynamics the delayed impact of an asset bubble on the financial network and mean-reversion features, as we explain in details in the following. The banks affect each other's robustness by being financially exposed to each other, for example because of cross-holdings, which results in a coupling of the drift terms. Following the setting in Battiston [6], we then assume that a fixed number of banks are directly investing in a bubble that affects their financial robustness. The remaining banks have the possibility to participate in the bubble by investing in the bubble banks. This results in a typical core/periphery structure for financial networks, where here the core is formed by the banks holding the bubble. In our model, banks' investments depend on the robustness of the other institutions, allowing for heterogeneity of the drift rates of the SDEs. More precisely, in our case the rates depend on the robustness of the attracting institution with a delay $\delta>0$, where the delay reflects the fact that the banks' investment do not immediately react to changes in the system. This extends previous models, where the coupling drift rates representing the weighted network connections are constant (as in [13], [17, [37, [55]), functions of time ([22], [58]) or of the difference in monetary reserves ([36]), but not functions of the state of other banks. In this way, we introduce a preferential attachment mechanism where the attractiveness of a node does not depend on its degree, but on its "fitness", as proposed by Bianconi and Barabàsi [11]. Due to this behaviour, the bubble 
causes a distortion in the network evolution: during the expanding phase of the bubble, the network structure shifts towards an increasingly intense and centralised connectivity due to the strong growth of the bubbly banks' robustness, which then causes instability in case the bubble bursts.

We then study the behaviour of the system when its size gets large. More precisely, we let the number of periphery banks tend to infinity, but keep the number of core banks holding the bubble constant and assume that their impact on the system does not vanish when the total number of banks tends to infinity. In this way the bubble produces a common stochastic source in the system that does not not average out even for large networks. Our main result then determines a partial mean-field limit for the system where the influence of the bubble is represented via stochastic interaction with the core banks even in the limit. Because of this term, the banks in the periphery are also affected by a potential bubble burst. This effect is amplified by the impossibility to immediately disinvest when the robustness of some banks decreases due to the delay $\delta$. We also refer to Chong and Klüppelberg [22] where the authors investigate partial mean-field limits in a different setting, without taking into account the delay and the influence of the bubble.

The remaining part of the paper is organised as follows. In Section 2 we introduce our model and some technical results. In Section 3 we define the limit system and prove a convergence result, whereas in Section 4 we perform Monte Carlo simulations both in the finite and in the limit systems in order to numerically investigate the impact of the bubble on systemic risk.

\section{The model}

Let $(\Omega, \mathcal{F}, \mathbb{F}, P)$ be a filtered probability space endowed with a $(m+n+2)$-dimensional Brownian motion $\bar{W}=\left(W_{t}^{1}, \ldots, W_{t}^{n}, W_{t}^{B, 1}, \ldots, W_{t}^{B, m}, B_{t}^{1}, B_{t}^{2}\right)_{t \geq 0}, m, n \in \mathbb{N}$, where $\mathbb{F}=$ $\left(\mathcal{F}_{t}\right)_{t \in \mathbb{R}^{+}}$is the natural filtration of $\bar{W}$. We consider a network of $m+n$ banks, consisting of $m$ banks holding a bubbly asset in their portfolio (also referred to as core), and $n$ banks that do not directly hold the bubbly asset (also referred to as periphery).

By following a similar approach as in Kley et al. [55], we model the robustness of the banks in the system. This coefficient dynamically evolves and represents a measure of how healthy a bank remains in stress situations. Let $\rho^{i, n}=\left(\rho_{t}^{i, n}\right)_{t \geq 0}, i=1, \ldots n$, and $\rho^{k, B}=\left(\rho_{t}^{k, B}\right)_{t \geq 0}$, $k=1, \ldots, m$, be the robustness of banks not holding and holding the bubble, respectively. We assume that they satisfy the following system of stochastic differential delay equations (SDDEs) for $t \geq \delta, \delta>0$,

$$
d \rho_{t}^{i, n}=\left(\frac{1}{n-1} \sum_{j=1, j \neq i}^{n} f^{P}\left(\rho_{t-\delta}^{j, n}-A_{t-\delta}^{n, m}\right)\left(\rho_{t}^{j, n}-A_{t}^{n, m}\right)+\frac{1}{m} \sum_{k=1}^{m} f^{B}\left(\rho_{t-\delta}^{k, B}-A_{t-\delta}^{n, m}\right)\left(\rho_{t}^{k, B}-A_{t}^{n, m}\right)\right) d t
$$




$$
\begin{aligned}
& +\lambda\left(A_{t}^{n, m}-\rho_{t}^{i, n}\right) d t+\sigma_{1} d W_{t}^{i} \\
d \rho_{t}^{k, B}= & \left(\frac{1}{n} \sum_{i=1}^{n} f^{P}\left(\rho_{t-\delta}^{i, n}-A_{t-\delta}^{n, m}\right)\left(\rho_{t}^{i, n}-A_{t}^{n, m}\right)+\frac{1}{m-1} \sum_{\ell=1, \ell \neq k}^{m} f^{B}\left(\rho_{t-\delta}^{\ell, B}-A_{t-\delta}^{n, m}\right)\left(\rho_{t}^{\ell, n}-A_{t}^{n, m}\right)\right) d t \\
& +\lambda\left(A_{t}^{n, m}-\rho_{t}^{k, B}\right) d t+\sigma_{2} d W_{t}^{k, B}+d \beta_{t}
\end{aligned}
$$

where $\lambda>0, \sigma_{1}>0, \sigma_{2}>0$ and

$$
A_{t}^{n, m}=\frac{1}{m+n}\left(\sum_{r=1}^{n} \rho_{t}^{r, n}+\sum_{h=1}^{m} \rho_{t}^{h, B}\right), \quad t \geq \delta,
$$

is the mean of the robustness of all the banks in the network at time $t$. For $t \in[0, \delta)$, we assume that $\left(\rho_{s}^{i, n}\right)_{s \in[0, \delta)},\left(\rho_{s}^{k, B}\right)_{s \in[0, \delta)}, i=1, \ldots, n, k=1, \ldots, m$, satisfy (2.1)-(2.2) with $\delta=0$, by following the approach of Mao [59]. We also suppose that $\rho_{0}^{i, n}=\rho_{0}>0$ for all $i=1, \ldots, n$.

Remark 1. Note that robustness may become negative in our model, as in [45] and [55], since it is used as an indicator of banks' creditworthiness. See also [37], where log-monetary reserves play the role of robustness.

The process $\beta=\left(\beta_{t}\right)_{t \geq 0}$ in 2.2 represents the influence of the asset price bubble on the robustness of core banks and has dynamics

$$
d \beta_{t}=\mu_{t} d t+\sigma_{t}^{B} d B_{t}^{1}, \quad t \geq 0
$$

where $\sigma^{B}=\left(\sigma_{t}^{B}\right)_{t \geq 0}$ is a positive and adapted process such that

$$
\int_{0}^{t} \mathbb{E}\left[\left|\sigma_{s}^{B}\right|^{2}\right] d s<\infty, \quad 0 \leq t<\infty
$$

and $\mu$ is an adapted process, unique strong solution of

$$
d \mu_{t}=\tilde{b}\left(\mu_{t}\right) d t+\tilde{\sigma}\left(\mu_{t}\right) d B_{t}^{2}, \quad t \geq 0
$$

where $\tilde{b}, \tilde{\sigma}$ fulfil the usual Lipschitz and sub-linear growth conditions such that there exists a unique solution of (2.6), satisfying

$$
\int_{0}^{t} \mathbb{E}\left[\left|\mu_{s}\right|^{2}\right] d s<\infty, \quad 0 \leq t<\infty
$$

In Section 4 we will specify a model for the bubbly evolution in (2.4) and provide further explanations on asset price bubbles, see Section 4.1.

We assume the following hypothesis on $f^{B}$ and $f^{P}$. 
Assumption 2.1. The functions $f^{B}, f^{P}: \mathbb{R} \rightarrow \mathbb{R}^{+}$are measurable, with

$$
f^{B}(0)=f^{B}\left(0^{+}\right)=f^{B}\left(0^{-}\right)<\infty, \quad f^{P}(0)=f^{P}\left(0^{+}\right)=f^{P}\left(0^{-}\right)<\infty,
$$

and such that the functions $F^{B}(x):=x f^{B}(x), F^{P}(x):=x f^{P}(x), x \in \mathbb{R}$, are Lipschitz continuous, i.e.

$$
\left|x f^{\ell}(x)-y f^{\ell}(y)\right| \leq K_{1}|x-y|, \quad x, y \in \mathbb{R}, \quad \ell=B, P, \quad K_{1}>0 .
$$

Note that 2.8) and (2.9) imply that $f^{B}$ and $f^{P}$ are continuous on $\mathbb{R}$ and bounded, since if $f(x) x$ is Lipschitz, then

$$
|f(x) x|=|f(x) x-f(0) \cdot 0| \leq K_{1}|x| .
$$

The interdependencies of the banks' robustness and corresponding contagion effects are specified through the drifts in (2.1) and (2.2). The term $\lambda\left(A_{t}^{n, m}-\rho_{t}^{i, n}\right)$ represents an attraction of the individual robustness towards the average robustness of the system with rate $\lambda$ as in the classical mean-field model (1.1). In addition to the homogeneous average term, we introduce the terms of type $f^{P}\left(\rho_{t-\delta}^{j, n}-A_{t-\delta}^{n, m}\right)\left(\rho_{t}^{j, n}-A_{t}^{n, m}\right)$ and $f^{B}\left(\rho_{t-\delta}^{k, B}-A_{t-\delta}^{n, m}\right)\left(\rho_{t}^{k, B}-A_{t}^{n, m}\right)$ that represent a robustness-dependent evolution of the network connectivity: for typically positive and increasing $f^{B}$ and $f^{P}$, bank $i$ is the more connected to bank $j$ the higher bank $j$ 's robustness is above the average. In this way, the evolution of the bubble alters the connectivity structure of the network according to a model of preferential attachment. Moreover, the propensity of a node $i$ to attract future links not only depends on the current level of robustness of $i$, but also on the robustness of the banks already connected to $i$. This produces different kinds of preferential attachment: a direct preferential attachment towards banks with the bubbly asset, and an indirect preferential attachment towards banks that have invested money in the banks with the bubbly asset, increasing their robustness. This mechanism is called preferential preferential attachment in Battiston [6], and creates a network with a set of financial institutions which are very strongly connected to each other. These banks form a cluster, which is in fact the core of the network. This is referred to as "strong clustering effect" in [6].

This change in network structure then comes along with an increasing systemic risk and instability in case the bubble burst, as noted by Battiston [6]. We introduce the delay $\delta>0$ to reflect the fact that the bank $i$ 's investment decisions does not immediately react to changes in bank $j$ 's robustness. Note that when there are no bubble banks and $f^{P}=\lambda$, the system (2.1)- (2.2) boils down to the basis mean-field model in (1.1), apart from the fact that here we have the term $\frac{1}{n-1}$ instead of $\frac{1}{n}$ in front of the sum, since we are averaging the investments with respect to the other $(n-1)$ banks. However, the impact of these terms is the same for large networks, i.e., when $n$ tends to infinity. 
Example 2.2. We have that $f(x)=1+2 \arctan (x) / \pi$ satisfies Assumption 2.1: $f$ takes values in [0,2], and both $f$ and $F(x)=x f(x)$ are Lipschitz, because they have bounded derivatives.

In particular, $f$ is increasing, so that if $\rho_{t}^{j}>\rho_{t}^{i}$ then the link towards $j$ is stronger than the link towards $i$. If the robustness $\rho_{t}^{j}$ of bank $j$ is equal to the average $A_{t}^{n, m}$ in (2.3), then the link towards bank $j$ has weight $f(0)=1$, if $\rho_{t}^{j}>A_{t}^{n, m}$ the link has weight bigger than 1 and if $\rho_{t}^{j}<A_{t}^{n, m}$ the link has weight less than 1 . If all the banks have the same robustness, we have an homogenous network, where all the links have weight equal to 1.

We note that different choices for $f$ are possible. In particular, we could use different functions for core and periphery banks (for example by considering a parametric dependence in $f$ ) in order to introduce heterogeneity in the model. Here we choose only one function for both kinds of banks for the sake of simplicity.

Furthermore, any constant function clearly satisfies Assumption 2.1. For such a choice, we have a static and homogenous network.

Proposition 2.3. Under Assumption 2.1, for every $\delta \geq 0$ there exists a unique strong solution for the system of SDEs (2.1)-2.2. Moreover,

$$
\begin{aligned}
& \sup _{0 \leq s \leq t} \mathbb{E}\left[\left|\rho_{s}^{i, n}\right|^{2}\right]<\infty, \quad 0<t<\infty, \quad i=1, \ldots, n, \\
& \sup _{0 \leq s \leq t} \mathbb{E}\left[\left|\rho_{s}^{k, B}\right|^{2}\right]<\infty, \quad 0<t<\infty, \quad k=1, \ldots, m .
\end{aligned}
$$

Proof. Suppose by simplicity $\lambda=1$ and that $\sigma^{B}=\left(\sigma_{t}^{B}\right)_{t \geq 0}$ is constant, i.e. $\sigma_{t}^{B}=\sigma_{B}>0$ for all $t \geq q^{1}$. For $\delta=0$ we can write the system of SDEs given by (2.1), 2.2) and (2.6) as an $(m+n+1)$-dimensional SDE

$$
d X_{t}=b\left(X_{t}\right) d t+\sigma\left(X_{t}\right) d \bar{W}_{t}, \quad t \geq 0
$$

where

$$
\bar{W}_{t}=\left(W_{t}^{1}, \ldots, W_{t}^{n}, W_{t}^{1, B}, \ldots, W_{t}^{m, B}, B_{t}^{1}, B_{t}^{2}\right)_{t \geq 0} .
$$

\footnotetext{
${ }^{1}$ We suppose that $\sigma^{B}=\left(\sigma_{t}^{B}\right)_{t \geq 0}$ is constant in order to ease the computations and the notation in the proof. However, condition 2.5 guarantees that the result also holds in more general cases.
} 
Moreover,

$$
b(x)=\left(\begin{array}{c}
\frac{1}{n-1} \sum_{j=2}^{n} f^{P}\left(x_{j}-\bar{x}\right)\left(x_{j}-\bar{x}\right)+\frac{1}{m} \sum_{k=n+1}^{m+n} f^{B}\left(x_{k}-\bar{x}\right)\left(x_{k}-\bar{x}\right)+\bar{x}-x_{1}, \\
\vdots \\
\frac{1}{n-1} \sum_{j=1}^{n-1} f^{P}\left(x_{j}-\bar{x}\right)\left(x_{j}-\bar{x}\right)+\frac{1}{m} \sum_{k=n+1}^{m+n} f^{B}\left(x_{k}-\bar{x}\right)\left(x_{k}-\bar{x}\right)+\bar{x}-x_{n} \\
\frac{1}{n} \sum_{j=1}^{n} f^{P}\left(x_{j}-\bar{x}\right)\left(x_{j}-\bar{x}\right)+\frac{1}{m-1} \sum_{k=n+2}^{m+n} f^{B}\left(x_{k}-\bar{x}\right)\left(x_{k}-\bar{x}\right)+\bar{x}-x_{n+1} \\
\vdots \\
\frac{1}{n} \sum_{j=1}^{n} f^{P}\left(x_{j}-\bar{x}\right)\left(x_{j}-\bar{x}\right)+\frac{1}{m-1} \sum_{k=n+1}^{m+n-1} f^{B}\left(x_{k}-\bar{x}\right)\left(x_{k}-\bar{x}\right)+\bar{x}-x_{m+n} \\
b\left(x_{m+n+1}\right)
\end{array}\right)
$$

with $x=\left(x_{1}, \ldots, x_{m+n+1}\right) \in \mathbb{R}^{m+n+1}$ and $\bar{x}=\frac{1}{m+n} \sum_{i=1}^{m+n} x_{i}$. Here $\sigma(x)$ is a $(m+n+1) \times$ $(m+n+1)$ block matrix of the form

$$
\sigma(x)=\left(\begin{array}{ccc}
\Sigma_{1} & 0 & 0 \\
0 & \Sigma_{2} & 0 \\
0 & 0 & \tilde{\sigma}\left(x_{m+n+1}\right)
\end{array}\right)
$$

where $\Sigma_{1}$ is a $n \times n$ diagonal matrix with diagonal $\left(\sigma_{1}, \ldots, \sigma_{1}\right)$ and $\Sigma_{2}$ is the $m \times(m+1)$ matrix

$$
\Sigma_{2}=\left(\begin{array}{ccccc}
\sigma_{2} & 0 & \ldots & 0 & \sigma_{B} \\
0 & \sigma_{2} & \ldots & 0 & \sigma_{B} \\
\vdots & \vdots & \ddots & 0 & \sigma_{B} \\
0 & 0 & \ldots & \sigma_{2} & \sigma_{B}
\end{array}\right)
$$

We use Theorem 2.9 in Chapter 5.2 of Karatzas and Shreve [54] to prove existence and uniqueness of the strong solution of (2.13), and that the second moments of the solution are finite, see the proof of Proposition 5.2.3 in Mazzon [60].

When $\delta>0$, equation 2.13 becomes

$$
d X_{t}=\bar{b}\left(X_{t}, X_{t-\delta}\right) d t+\bar{\sigma}\left(X_{t}, X_{t-\delta}\right) d \bar{W}_{t}, \quad t \geq \delta
$$

where $\bar{\sigma}(x, y)=\sigma(x)$ as in 2.15 and

$$
b(x, y)=\left(\begin{array}{c}
\frac{1}{n-1} \sum_{j=2}^{n} f^{P}\left(y_{j}-\bar{y}\right)\left(x_{j}-\bar{x}\right)+\frac{1}{m} \sum_{k=n+1}^{m+n} f^{B}\left(y_{k}-\bar{y}\right)\left(x_{k}-\bar{x}\right)+\bar{x}-x_{1}, \\
\vdots \\
\frac{1}{n-1} \sum_{j=1}^{n-1} f^{P}\left(y_{j}-\bar{y}\right)\left(x_{j}-\bar{x}\right)+\frac{1}{m} \sum_{k=n+1}^{m+n} f^{B}\left(y_{k}-\bar{y}\right)\left(x_{k}-\bar{x}\right)+\bar{x}-x_{n} \\
\frac{1}{n} \sum_{j=1}^{n} f^{P}\left(y_{j}-\bar{y}\right)\left(x_{j}-\bar{x}\right)+\frac{1}{m-1} \sum_{k=n+2}^{m+n} f^{B}\left(y_{k}-\bar{y}\right)\left(x_{k}-\bar{x}\right)+\bar{x}-x_{n+1} \\
\vdots \\
y \frac{1}{n} \sum_{j=1}^{n} f^{P}\left(y_{j}-\bar{y}\right)\left(x_{j}-\bar{x}\right)+\frac{1}{m-1} \sum_{k=n+1}^{m+n-1} f^{B}\left(y_{k}-\bar{y}\right)\left(x_{k}-\bar{x}\right)+\bar{x}-x_{m+n} \\
b\left(x_{m+n+1}\right)
\end{array}\right) .
$$


By Theorem 3.1 in Mao [59, chapter 5], to prove existence and uniqueness of the solution it suffices to show that the linear growth condition

$$
\|\bar{b}(x, y)\|^{2} \leq C\left(1+\|x\|^{2}+\|y\|^{2}\right)
$$

holds and that $\bar{b}$ is Lipschitz in the variable $x$ uniformly in $y$, i.e. that there exists a constant $\tilde{K} \in(0, \infty)$ such that

$$
\left\|\bar{b}(x, y)-\bar{b}\left(x^{\prime}, y\right)\right\|^{2} \leq \tilde{K}\left\|x-x^{\prime}\right\|^{2}
$$

for all $y \in \mathbb{R}, x, x^{\prime} \in \mathbb{R}^{m+n}$. Property 2.17) can be proven by computations similar to the ones used for $\delta=0$, see the proof of Proposition 5.2.3 in [60]. For the Lipschitz condition we have

$$
\begin{aligned}
\left|\bar{b}_{1}(x, y)-\bar{b}_{1}\left(x^{\prime}, y\right)\right| \leq & \frac{1}{n-1} \sum_{j=2}^{n}\left|f^{P}\left(y_{j}-\bar{y}\right)\right|\left|\left(x_{j}-\bar{x}\right)-\left(x_{j}^{\prime}-\bar{x}^{\prime}\right)\right| \\
& +\frac{1}{m} \sum_{k=n+1}^{m+n}\left|f^{B}\left(y_{k}-\bar{y}\right)\right|\left|\left(x_{k}-\bar{x}\right)-\left(x_{k}^{\prime}-\bar{x}^{\prime}\right)\right|+\left|\bar{x}-\bar{x}^{\prime}\right|+\left|x_{1}-x_{1}^{\prime}\right| .
\end{aligned}
$$

Hence, as $f^{B}$ and $f^{P}$ are bounded by $K_{1}$, the computations to show 2.18 are identical to the case for $\delta=0$.

In order to prove (2.11) and 2.12, we apply the same argument used in the proof of Theorem 3.1 in Mao [59, chapter 5]: on $[0, \delta]$ we have by hypothesis a classic stochastic differential equation, and by Theorem 2.9 in Chapter 5.2 of [54]

$$
\mathbb{E}\left[\sup _{0 \leq s \leq \delta}\left\|X_{s}\right\|^{2}\right]<\infty
$$

On the interval $[\delta, 2 \delta]$, we can write equation (2.16) as

$$
d X_{t}=\bar{b}\left(X_{t}, \xi_{t}\right) d t+\bar{\sigma}\left(X_{t}, \xi_{t}\right) d W_{t}, \quad \delta \leq t \leq 2 \delta
$$

where $\xi_{t}=X_{t-\delta}$. Once the solution on $[0, \delta]$ is known, this is again a classic SDE (without delay) with initial value $X_{\delta}=\xi_{0}$, so that again by Theorem 2.9 in Chapter 5.2 of [54], there exists a constant $C_{2 \delta}>0$ such that

$$
\mathbb{E}\left[\sup _{\delta \leq s \leq 2 \delta}\left\|X_{s}\right\|^{2}\right] \leq C_{2 \delta}\left(1+\mathbb{E}\left[\left\|X_{\delta}\right\|^{2}\right]\right) e^{2 \delta C_{2 \delta}}
$$

which is finite by $(2.19)$. Repeating this argument on the interval $[2 \delta, 3 \delta]$, we obtain

$$
\mathbb{E}\left[\sup _{2 \delta \leq s \leq 3 \delta}\left\|X_{s}\right\|^{2}\right] \leq C_{3 \delta}\left(1+\mathbb{E}\left[\left\|X_{2 \delta}\right\|^{2}\right]\right) e^{3 \delta C_{3 \delta}} \leq C_{3 \delta}\left(1+\mathbb{E}\left[\sup _{\delta \leq s \leq 2 \delta}\left\|X_{s}\right\|^{2}\right]\right) e^{3 \delta C_{3 \delta}}<\infty
$$


by 2.20 . Recursively we have

$$
\mathbb{E}\left[\sup _{(k-1) \delta \leq s \leq k \delta}\left\|X_{s}\right\|^{2}\right]<\infty
$$

Then,

$$
\sup _{0 \leq s \leq t} \mathbb{E}\left[\left\|X_{s}\right\|^{2}\right]=\sup _{s \in[\bar{k} \delta,(\bar{k}+1) \delta]} \mathbb{E}\left[\left\|X_{s}\right\|^{2}\right]<\infty,
$$

for some $\bar{k}$ with $[\bar{k} \delta,(\bar{k}+1) \delta] \subseteq[0, t]$.

\section{Mean field limit}

We now study a mean field limit for the system of banks 2.1)-2.2 for large $n$.

Define the processes $\tilde{\rho}^{i}=\left(\tilde{\rho}_{t}^{i}\right)_{t \geq 0}, i=1, \ldots, n, \bar{\rho}^{k, B}=\left(\bar{\rho}_{t}^{k, B}\right)_{t \geq 0}, k=1, \ldots, m$, and $\nu=\left(\nu_{t}\right)_{t \geq 0}$ as the solutions of the following system of SDEs for $t \geq \delta$ :

$$
\begin{aligned}
d \tilde{\rho}_{t}^{i}= & -\lambda \tilde{\rho}_{t}^{i} d t+\sigma_{1} d W_{t}^{i} \\
d \nu_{t}= & \left(\varphi(t, t-\delta)+\frac{1}{m} \sum_{k=1}^{m} f^{B}\left(\bar{\rho}_{t-\delta}^{k, B}-\nu_{t-\delta}-\mathbb{E}\left[\tilde{\rho}_{t-\delta}^{i}\right]\right)\left(\bar{\rho}_{t}^{k, B}-\nu_{t}-\mathbb{E}\left[\tilde{\rho}_{t}^{i}\right]\right)+\lambda \mathbb{E}\left[\tilde{\rho}_{t}^{i}\right]\right) d t \\
d \bar{\rho}_{t}^{k, B}= & \left(\varphi(t, t-\delta)+\frac{1}{m-1} \sum_{\ell=1, \ell \neq k}^{m} f^{B}\left(\bar{\rho}_{t-\delta}^{\ell, B}-\nu_{t-\delta}-\mathbb{E}\left[\tilde{\rho}_{t-\delta}^{i}\right]\right)\left(\bar{\rho}_{t}^{\ell, B}-\nu_{t}-\mathbb{E}\left[\tilde{\rho}_{t}^{i}\right]\right)\right) d t \\
& +\left(\mu_{t}+\lambda\left(\mathbb{E}\left[\tilde{\rho}_{t}^{i}\right]+\nu_{t}-\bar{\rho}_{t}^{k, B}\right)\right) d t+\sigma_{2} d W_{t}^{k, B}+\sigma_{t}^{B} d B_{t}^{1},
\end{aligned}
$$

with

$$
\begin{aligned}
\varphi(t, t-\delta) & :=\mathbb{E}\left[f^{P}\left(\tilde{\rho}_{t-\delta}^{i}-\mathbb{E}\left[\tilde{\rho}_{t-\delta}^{i}\right]\right)\left(\tilde{\rho}_{t}^{i}-\mathbb{E}\left[\tilde{\rho}_{t}^{i}\right]\right)\right] \\
& =\mathbb{E}\left[\mathbb{E}\left[f^{P}\left(\tilde{\rho}_{t-\delta}^{i}-\mathbb{E}\left[\tilde{\rho}_{t-\delta}^{i}\right]\right)\left(\tilde{\rho}_{t}^{i}-\mathbb{E}\left[\tilde{\rho}_{t}^{i}\right]\right) \mid \tilde{\rho}_{t-\delta}^{i}\right]\right] \\
& =\mathbb{E}\left[f^{P}\left(\tilde{\rho}_{t-\delta}^{i}-\mathbb{E}\left[\tilde{\rho}_{t-\delta}^{i}\right]\right) \mathbb{E}\left[\tilde{\rho}_{t}^{i} \mid \tilde{\rho}_{t-\delta}^{i}\right]\right]-\mathbb{E}\left[\tilde{\rho}_{t}^{i}\right] \mathbb{E}\left[f^{P}\left(\tilde{\rho}_{t-\delta}^{i}-\mathbb{E}\left[\tilde{\rho}_{t-\delta}^{i}\right]\right)\right] \\
& =e^{-\lambda \delta} \mathbb{E}\left[f^{P}\left(\tilde{\rho}_{t-\delta}^{i}-\mathbb{E}\left[\tilde{\rho}_{t-\delta}^{i}\right]\right) \tilde{\rho}_{t-\delta}^{i}\right]-\rho_{0} e^{-\lambda t} \mathbb{E}\left[f^{P}\left(\tilde{\rho}_{t-\delta}^{i}-\mathbb{E}\left[\tilde{\rho}_{t-\delta}^{i}\right]\right)\right],
\end{aligned}
$$

for $t \geq \delta$. For $t \in[0, \delta]$ we assume that $\left(\tilde{\rho}_{t}\right)_{0 \leq t \leq \delta},\left(\nu_{t}\right)_{0 \leq t \leq \delta}$ and $\left(\bar{\rho}_{t}^{k, B}\right)_{0 \leq t \leq \delta}$ satisfy (3.1)-(3.3) for $\delta=0$, with initial conditions $\tilde{\rho}_{0}^{i}=\rho_{0} \in \mathbb{R}, \nu_{0}=0, \bar{\rho}_{0}^{k, B}=\rho_{0}^{k, B} \in \mathbb{R}$.

Note that in equation (3.2) the expression of $\varphi$ is independent of the choice of $i$ since $\tilde{\rho}^{i}$, $i=1, \ldots, n$, are identically distributed. For the same reason, the process $\nu$ in $(3.2)$ does not depend on $i$.

Set

$$
\bar{\rho}^{i}:=\tilde{\rho}^{i}+\nu, \quad i=1, \ldots, n .
$$


In particular,

$$
\begin{aligned}
\bar{\rho}_{t}^{i}= & \bar{\rho}_{\delta}^{i}+\int_{\delta}^{t}\left(\varphi(s, s-\delta)+\frac{1}{m} \sum_{k=1}^{m} f^{B}\left(\bar{\rho}_{s-\delta}^{k, B}-\nu_{s-\delta}-\mathbb{E}\left[\tilde{\rho}_{s-\delta}^{i}\right]\right)\left(\bar{\rho}_{s}^{k, B}-\nu_{s}-\mathbb{E}\left[\tilde{\rho}_{s}^{i}\right]\right)+\lambda\left(\mathbb{E}\left[\tilde{\rho}_{s}^{i}\right]-\tilde{\rho}_{s}^{i}\right)\right) d s \\
& +\sigma_{1} W_{s}^{i}, \quad t \geq \delta .
\end{aligned}
$$

Remark 2. The processes $\left(\bar{\rho}_{t}^{i}\right)_{t \geq 0}, i=1, \ldots, n$, are not independent, so a priori the strong law of large numbers could not be applied. However, as shown in (3.5), $\bar{\rho}^{i}$ can be written as the sum of $\left(\tilde{\rho}_{t}^{i}\right)_{t \geq 0}$ from (3.1) and $\left(\nu_{t}\right)_{t \geq 0}$ from (3.2), respectively. In particular, the processes $\tilde{\rho}^{i}, i=1, \ldots, n$, are independent Ornstein-Uhlenbeck processes, and $\nu$ is independent of $i$ and common to all $\bar{\rho}^{i}, i=1, \ldots, n$. In this way, we obtain a decomposition of $\bar{\rho}^{i}$ which permits to apply the strong law of large numbers to the sum of $\tilde{\rho}^{i}, i=1, \ldots, n$, and then prove Theorem 3.2 .

Proposition 3.1. Under Assumption 2.1, for every $\delta \geq 0$ there exists a unique strong solution of the system of SDEs (3.1)-(3.3). In particular,

$$
\begin{aligned}
& \sup _{0 \leq s \leq t} \mathbb{E}\left[\left|\nu_{s}\right|^{2}\right]<\infty, \quad 0<t<\infty, \\
& \sup _{0 \leq s \leq t} \mathbb{E}\left[\left|\rho_{s}^{k, B}\right|^{2}\right]<\infty, \quad 0<t<\infty, \quad k=1, \ldots, m .
\end{aligned}
$$

Proof. For the sake of simplicity we take $\lambda=1$ and $\sigma_{t}^{B}=\sigma_{B}>0$ for all $t \geq 0$ as before. It is well known that (3.1) admits a unique strong solution. For $\delta=0$, the system given by (3.2), (3.3) and (2.6) can be written as an $(m+2)$-dimensional SDE

$$
d X_{t}=b\left(t, X_{t}\right) d t+\sigma\left(t, X_{t}\right) d W_{t}, \quad t \geq 0,
$$

where $W=\left(W_{t}^{B, 1}, \ldots, W_{t}^{B, m}, B_{t}^{1}, B_{t}^{2}\right)_{t \geq 0}$, and

$$
b(t, x)=\left(\begin{array}{c}
\varphi(t)+\frac{1}{m} \sum_{k=1}^{m} f^{B}\left(x_{k}-x_{1}-\psi(t)\right)\left(x_{k}-x_{1}-\psi(t)\right)+\psi(t), \\
\varphi(t)+\frac{1}{m-1} \sum_{\ell=3}^{m+1} f^{B}\left(x_{\ell}-x_{1}-\psi(t)\right)\left(x_{\ell}-x_{1}-\psi(t)\right)+x_{1}+x_{m+2}-x_{2}+\psi(t), \\
\vdots \\
\varphi(t)+\frac{1}{m-1} \sum_{\ell=2}^{m} f^{B}\left(x_{\ell}-x_{1}-\psi(t)\right)\left(x_{\ell}-x_{1}-\psi(t)\right)+x_{1}+x_{m+2}-x_{m+1}+\psi(t), \\
\tilde{b}\left(x_{m+2}\right)
\end{array}\right)
$$

with $\psi(t)=\mathbb{E}\left[\tilde{\rho}_{t}^{i}\right]$ and

$$
\varphi(t):=\mathbb{E}\left[f^{P}\left(\tilde{\rho}_{t}^{i}-\mathbb{E}\left[\tilde{\rho}_{t}^{i}\right]\right)\left(\tilde{\rho}_{t}^{i}-\mathbb{E}\left[\tilde{\rho}_{t}^{i}\right]\right)\right], \quad t \geq 0
$$


The $(m+2) \times(m+2)$ matrix $\sigma(x)$ has the form

$$
\sigma(t, x)=\left(\begin{array}{cccccc}
0 & 0 & \ldots & 0 & 0 & 0 \\
\sigma_{2} & 0 & \ldots & 0 & \sigma_{B} & 0 \\
0 & \sigma_{2} & \ldots & 0 & \sigma_{B} & 0 \\
\vdots & \vdots & \ddots & 0 & \sigma_{B} & 0 \\
0 & 0 & \ldots & \sigma_{2} & \sigma_{B} & 0 \\
0 & 0 & \ldots & 0 & 0 & \tilde{\sigma}\left(x_{m+2}\right)
\end{array}\right)
$$

Computations similar to the ones in Proposition 2.3 guarantee existence and uniqueness of the solution of $(3.9)$ and that the second moments exist finite by Theorem 2.9 in Chapter 5.2 of [54], see 60].

The proof for the case $\delta>0$, based on Theorem 3.1 in Mao [59, chapter 5], is analogous to the one of Proposition 2.3 .

We now present the main theoretical result of the paper, which guarantees that, in the setting of Assumption 2.1, the system (2.1), 2.2) can be approximated by (3.6), (3.3) for large networks. Denote $|x-y|_{t}^{*}=\sup _{s \leq t}\left|x_{s}-y_{s}\right|$. We have the following

Theorem 3.2. Fix $i \in \mathbb{N}$. Under Assumption 2.1, for any $t \in[0, \infty)$ and $\delta \geq 0$ it holds

$$
\lim _{n \rightarrow \infty}\left(\mathbb{E}\left[\left|\rho^{i, n}-\bar{\rho}^{i}\right|_{t}^{*}\right]+\mathbb{E}\left[\left|\rho^{k, B}-\bar{\rho}^{k, B}\right|_{t}^{*}\right]\right)=0, \quad k=1, \ldots, m
$$

where $\rho^{i, n}, \bar{\rho}^{i}, \rho^{k, B}, \bar{\rho}^{k, B}$ are defined in 2.1), (3.6), 2.2), 3.3 respectively.

Remark 3. We now interpret the results of Theorem 3.2. Note that the influence of the bubble on the limit system is twofold: it rules out propagation of chaos and increases systemic risk. Indeed, the bubble makes the banks of the core mutually dependent at the limit, as they share a common stochastic source. Furthermore, their impact does not vanish in the limit. As a consequence, all banks in the system remain dependent on each other in large networks as well. This breaks down the propagation of chaos, that is the property by which the system decouples more and more as the network gets larger. On the contrary, the bubble acts as a driving force of the system in the limit, too.

The term

$$
\frac{1}{m} \sum_{k=1}^{m} f^{B}\left(\bar{\rho}_{t-\delta}^{k, B}-\nu_{t-\delta}-\mathbb{E}\left[\tilde{\rho}_{t-\delta}^{i}\right]\right)\left(\bar{\rho}_{t}^{k, B}-\nu_{t}-\mathbb{E}\left[\tilde{\rho}_{t}^{i}\right]\right)
$$

in (3.2) makes this influence explicit: also the banks not holding the bubble are affected by its evolution, through the robustness of the banks with the bubbly asset. Moreover, again by (3.13) it can be seen how the bubble increases systemic risk: when the bubble bursts, also the banks in the periphery suffer a loss, because they are not able to promptly disinvest 
due to the delay $\delta$ in 3.13 . In this way the most systemic banks (i.e., the most connected institutions in the network) are the most exposed to the shock as well.

We also note that the mean reverting term $\lambda\left(\mathbb{E}\left[\tilde{\rho}_{t}^{i}\right]+\nu_{t}-\bar{\rho}_{t}^{k, B}\right)$ in $(3.3)$, which is the limit of $\lambda\left(A_{t}^{n, m}-\rho_{t}^{k, B}\right)$ in $(2.2)$, reduces the risk since it slows down the fall of $\bar{\rho}^{k, B}$ after the burst.

For further details, we refer to Section 4.3 , where numerical simulations are performed in order to investigate the behaviour of the system after the burst of the bubble.

We provide the proof of Theorem 3.2 in the Appendix. In order to prove Theorem 3.2 , we give the following

Proposition 3.3. Under Assumption 2.1, for $0 \leq \delta<\infty$,

$\lim _{n \rightarrow \infty} \int_{0}^{\delta} \mathbb{E}\left[\left\lfloor\frac{1}{n} \sum_{i=1}^{n} f^{P}\left(\bar{\rho}_{s}^{i}-\bar{A}_{s}^{n, m}\right)\left(\bar{\rho}_{s}^{i}-\bar{A}_{s}^{n, m}\right)-\mathbb{E}\left[f^{P}\left(\tilde{\rho}_{s}^{i}-\mathbb{E}\left[\tilde{\rho}_{s}^{i}\right]\right)\left(\tilde{\rho}_{s}^{i}-\mathbb{E}\left[\tilde{\rho}_{s}^{i}\right]\right)\right]\right\rfloor d s=0\right.$,

and

$\lim _{n \rightarrow \infty} \int_{\delta}^{t} \mathbb{E}\left[\left|\frac{1}{n} \sum_{i=1}^{n} f^{P}\left(\bar{\rho}_{s-\delta}^{i}-\bar{A}_{s-\delta}^{n, m}\right)\left(\bar{\rho}_{s}^{i}-\bar{A}_{s}^{n, m}\right)-\mathbb{E}\left[f^{P}\left(\tilde{\rho}_{s-\delta}^{i}-\mathbb{E}\left[\tilde{\rho}_{s-\delta}^{i}\right]\right)\left(\tilde{\rho}_{s}^{i}-\mathbb{E}\left[\tilde{\rho}_{s}^{i}\right]\right)\right]\right|\right] d s=0$,

for $0 \leq \delta \leq t<\infty$, where $\tilde{\rho}^{i}$ and $\bar{\rho}^{i}$ satisfy (3.1) and (3.6), respectively, and

$$
\bar{A}_{t}^{n, m}=\frac{1}{m+n}\left(\sum_{r=1}^{n} \bar{\rho}_{t}^{r}+\sum_{h=1}^{m} \bar{\rho}_{t}^{h, B}\right), \quad t \geq 0 .
$$

Proof. We limit ourselves to prove the second limit, since the first one follows as a particular case. Let us write, for $t \geq \delta>0$,

$$
\begin{aligned}
& \mathbb{E}[\left.\left|\frac{1}{n} \sum_{i=1}^{n} f^{P}\left(\bar{\rho}_{t-\delta}^{i}-\bar{A}_{t-\delta}^{n, m}\right)\left(\bar{\rho}_{t}^{i}-\bar{A}_{t}^{n, m}\right)-\mathbb{E}\left[f^{P}\left(\tilde{\rho}_{t-\delta}^{i}-\mathbb{E}\left[\tilde{\rho}_{t-\delta}^{i}\right]\right)\left(\tilde{\rho}_{t}^{i}-\mathbb{E}\left[\tilde{\rho}_{t}^{i}\right]\right)\right]\right|\right] \\
& \leq \frac{1}{n} \sum_{i=1}^{n} \mathbb{E}\left[\left|f^{P}\left(\bar{\rho}_{t-\delta}^{i}-\bar{A}_{t-\delta}^{n, m}\right)\left(\bar{\rho}_{t}^{i}-\bar{A}_{t}^{n, m}\right)-f^{P}\left(\tilde{\rho}_{t-\delta}^{i}-\mathbb{E}\left[\tilde{\rho}_{t-\delta}^{i}\right]\right)\left(\tilde{\rho}_{t}^{i}-\mathbb{E}\left[\tilde{\rho}_{t}^{i}\right]\right)\right|\right] \\
& \quad+\mathbb{E}\left[\left|\frac{1}{n} \sum_{i=1}^{n} f^{P}\left(\tilde{\rho}_{t-\delta}^{i}-\mathbb{E}\left[\tilde{\rho}_{t-\delta}^{i}\right]\right)\left(\tilde{\rho}_{t}^{i}-\mathbb{E}\left[\tilde{\rho}_{t}^{i}\right]\right)-\mathbb{E}\left[f^{P}\left(\tilde{\rho}_{t-\delta}^{i}-\mathbb{E}\left[\tilde{\rho}_{t-\delta}^{i}\right]\right)\left(\tilde{\rho}_{t}^{i}-\mathbb{E}\left[\tilde{\rho}_{t}^{i}\right]\right)\right]\right|\right],
\end{aligned}
$$

since $\bar{\rho}^{i}, i=1, \ldots, n$ are identically distributed and the same holds for $\tilde{\rho}^{i}, i=1, \ldots, n$. By (3.5) we have that

$$
\bar{A}_{t}^{n, m}=\frac{1}{m+n}\left(\sum_{r=1}^{n} \bar{\rho}_{t}^{r}+\sum_{h=1}^{m} \bar{\rho}_{t}^{h, B}\right)=\frac{1}{m+n}\left(n \nu_{t}+\sum_{r=1}^{n} \tilde{\rho}_{t}^{r}+\sum_{h=1}^{m} \bar{\rho}_{t}^{h, B}\right),
$$


so that

$$
\lim _{n \rightarrow \infty} \bar{A}_{t}^{n, m}=\nu_{t}+\lim _{n \rightarrow \infty} \frac{1}{m+n} \sum_{r=1}^{n} \tilde{\rho}_{t}^{r}=\nu_{t}+\mathbb{E}\left[\tilde{\rho}_{t}^{i}\right], \quad \text { a.s. },
$$

by 2.12 and the strong law of large numbers, as $\tilde{\rho}^{i}, i=1, \ldots, n$, are independent and identically distributed. Then we have

$$
\begin{aligned}
\lim _{n \rightarrow \infty} f^{P}\left(\bar{\rho}_{t-\delta}^{i}-\bar{A}_{t-\delta}^{n, m}\right)\left(\bar{\rho}_{t}^{i}-\bar{A}_{t}^{n, m}\right)= & f^{P}\left(\nu_{t-\delta}+\tilde{\rho}_{t-\delta}^{i}-\left(\nu_{t-\delta}+\mathbb{E}\left[\tilde{\rho}_{t-\delta}^{i}\right]\right)\right)\left(\nu_{t}+\tilde{\rho}_{t}^{i}-\left(\nu_{t}+\mathbb{E}\left[\tilde{\rho}_{t}^{i}\right]\right)\right) \\
& =f^{P}\left(\tilde{\rho}_{t-\delta}^{i}-\mathbb{E}\left[\tilde{\rho}_{t-\delta}^{i}\right]\right)\left(\tilde{\rho}_{t}^{i}-\mathbb{E}\left[\tilde{\rho}_{t}^{i}\right]\right) \quad \text { a.s. }
\end{aligned}
$$

We now prove that the family of random variables $\left\{\frac{1}{n} \sum_{i=1}^{n} f^{P}\left(\bar{\rho}_{s-\delta}^{i}-\bar{A}_{s-\delta}^{n, m}\right)\left(\bar{\rho}_{s}^{i}-\bar{A}_{s}^{n, m}\right)\right\}_{n \in \mathbb{N}}$ is uniformly integrable for every $s \in[\delta, t]$, so that convergence almost surely implies convergence in $L^{1}$.

By point (iii) of Theorem 11 in Protter [66, chapter 1] it is enough to prove that for every $s \in[\delta, t]$,

$$
\sup _{n} \mathbb{E}\left[\left(\frac{1}{n} \sum_{i=1}^{n} f^{P}\left(\bar{\rho}_{s-\delta}^{i}-\bar{A}_{s-\delta}^{n, m}\right)\left(\bar{\rho}_{s}^{i}-\bar{A}_{s}^{n, m}\right)\right)^{2}\right]<\infty .
$$

For every $s \in[\delta, t]$, we have that

$$
\begin{aligned}
\mathbb{E}[ & \left.\left(\frac{1}{n} \sum_{i=1}^{n} f^{P}\left(\bar{\rho}_{s-\delta}^{i}-\bar{A}_{s-\delta}^{n, m}\right)\left(\bar{\rho}_{s}^{i}-\bar{A}_{s}^{n, m}\right)\right)^{2}\right] \leq\left(K_{1}\right)^{2} \mathbb{E}\left[\left(\frac{1}{n} \sum_{i=1}^{n}\left|\bar{\rho}_{s}^{i}-\bar{A}_{s}^{n, m}\right|\right)^{2}\right] \\
& \leq\left(K_{1}\right)^{2} \mathbb{E}\left[\left((1-n /(m+n))\left|\nu_{s}\right|+\left|\tilde{\rho}_{s}^{i}\right|+\frac{1}{m+n} \sum_{r=1}^{n}\left|\tilde{\rho}_{s}^{r}\right|+\frac{1}{m+n} \sum_{h=1}^{m}\left|\bar{\rho}_{s}^{h, B}\right|\right)^{2}\right] \\
& \leq\left(K_{1}\right)^{2} \mathbb{E}\left[\left(\left|\nu_{s}\right|+\left|\tilde{\rho}_{s}^{i}\right|+\frac{1}{n} \sum_{r=1}^{n}\left|\tilde{\rho}_{s}^{r}\right|+\frac{1}{m} \sum_{h=1}^{m}\left|\bar{\rho}_{s}^{h, B}\right|\right)^{2}\right] \\
& \leq 4\left(K_{1}\right)^{2}\left(\mathbb{E}\left[\left|\nu_{s}\right|^{2}+\left|\tilde{\rho}_{s}^{i}\right|^{2}+\sum_{k=1}^{m}\left|\bar{\rho}_{s}^{k, B}\right|^{2}\right]+\mathbb{E}\left[\left(\frac{1}{n} \sum_{r=1}^{n}\left|\tilde{\rho}_{s}^{r}\right|\right)^{2}\right]\right) \\
& \leq 4\left(K_{1}\right)^{2}\left(\mathbb{E}\left[\left|\nu_{s}\right|^{2}+\left|\tilde{\rho}_{s}^{i}\right|^{2}+\sum_{k=1}^{m}\left|\bar{\rho}_{s}^{k, B}\right|^{2}\right]+\frac{1}{n} \mathbb{E}\left[\sum_{r=1}^{n}\left|\tilde{\rho}_{s}^{r}\right|^{2}\right]\right) . \\
& \leq 4\left(K_{1}\right)^{2}\left(\mathbb{E}\left[\left|\nu_{s}\right|^{2}+\left|\tilde{\rho}_{s}^{i}\right|^{2}+\sum_{k=1}^{m}\left|\bar{\rho}_{s}^{k, B}\right|^{2}\right]+\mathbb{E}\left[\left|\tilde{\rho}_{s}^{i}\right|^{2}\right]\right)<\infty,
\end{aligned}
$$

by $(3.7)$ and $(3.8)$ and because $\left.\mathbb{E}\left|\tilde{\rho}_{s}^{i}\right|^{2}\right]<\infty$. Hence, $\left\{\frac{1}{n} \sum_{i=1}^{n} f^{P}\left(\bar{\rho}_{s-\delta}^{i}-\bar{A}_{s-\delta}^{n, m}\right)\left(\bar{\rho}_{s}^{i}-\bar{A}_{s}^{n, m}\right)\right\}_{n \in \mathbb{N}}$ is uniformly integrable and we obtain therefore by (3.16) that

$$
\lim _{n \rightarrow \infty} \mathbb{E}\left[\left|f^{P}\left(\bar{\rho}_{t-\delta}^{i}-\bar{A}_{t-\delta}^{n, m}\right)\left(\bar{\rho}_{t}^{i}-\bar{A}_{t}^{n, m}\right)-f^{P}\left(\tilde{\rho}_{t-\delta}^{i}-\mathbb{E}\left[\tilde{\rho}_{t-\delta}^{i}\right]\right)\left(\tilde{\rho}_{t}^{i}-\mathbb{E}\left[\tilde{\rho}_{t}^{i}\right]\right)\right|\right]=0
$$


Moreover, for $\delta \leq s \leq t$,

$$
\begin{aligned}
& \mathbb{E}\left[\left|f^{P}\left(\bar{\rho}_{s-\delta}^{i}-\bar{A}_{s-\delta}^{n, m}\right)\left(\bar{\rho}_{s}^{i}-\bar{A}_{s}^{n, m}\right)-f^{P}\left(\tilde{\rho}_{s-\delta}^{i}-\mathbb{E}\left[\tilde{\rho}_{s-\delta}^{i}\right]\right)\left(\tilde{\rho}_{s}^{i}-\mathbb{E}\left[\tilde{\rho}_{s}^{i}\right]\right)\right|\right] \\
& \quad \leq K_{1}\left(\mathbb{E}\left[\left|\bar{\rho}_{s}^{i}-\bar{A}_{s}^{n, m}\right|\right]+\mathbb{E}\left[\mid \tilde{\rho}_{s}^{i}-\mathbb{E}\left[\tilde{\rho}_{s}^{i}\right]\right]\right),
\end{aligned}
$$

where the second term belongs to $L^{1}([\delta, t])$ and does not depend on $n$. On the other hand, we have

$$
\begin{aligned}
\int_{0}^{t} \mathbb{E}\left[\left|\bar{\rho}_{s}^{i}-\bar{A}_{s}^{n, m}\right|\right] d s & \leq \int_{0}^{t} \mathbb{E}\left[\left|\tilde{\rho}_{s}^{i}\right|+(1-n /(m+n))\left|\nu_{s}\right|+\frac{1}{m+n} \sum_{r=1}^{n}\left|\tilde{\rho}_{s}^{r}\right|+\frac{1}{m+n} \sum_{h=1}^{m}\left|\bar{\rho}_{s}^{h, B}\right|\right] d s \\
& \leq \int_{0}^{t} \mathbb{E}\left[2\left|\tilde{\rho}_{s}^{i}\right|+\left|\nu_{s}\right|+\left|\bar{\rho}_{s}^{h, B}\right|\right] d s \\
& \leq t \sup _{0 \leq s \leq t} \mathbb{E}\left[2\left|\tilde{\rho}_{s}^{i}\right|+\left|\nu_{s}\right|+\left|\bar{\rho}_{s}^{h, B}\right|\right]<\infty,
\end{aligned}
$$

by (3.7) and (3.8). We can then apply the dominated convergence theorem to obtain, for $t \in[\delta, \infty)$,

$$
\lim _{n \rightarrow \infty} \int_{\delta}^{t} \mathbb{E}\left[\left|f^{P}\left(\bar{\rho}_{s-\delta}^{i}-\bar{A}_{s-\delta}^{n, m}\right)\left(\bar{\rho}_{s}^{i}-\bar{A}_{s}^{n, m}\right)-f^{P}\left(\tilde{\rho}_{s-\delta}^{i}-\mathbb{E}\left[\tilde{\rho}_{s-\delta}^{i}\right]\right)\left(\tilde{\rho}_{s}^{i}-\mathbb{E}\left[\tilde{\rho}_{s}^{i}\right]\right)\right|\right] d s=0, \quad t \geq \delta .
$$

It remains to show that for $t \geq \delta$ it holds

$$
\lim _{n \rightarrow \infty} \int_{\delta}^{t} \mathbb{E}\left[\left\lfloor\frac{1}{n} \sum_{i=1}^{n} f^{P}\left(\tilde{\rho}_{s-\delta}^{i}-\mathbb{E}\left[\tilde{\rho}_{s-\delta}^{i}\right]\right)\left(\tilde{\rho}_{s}^{i}-\mathbb{E}\left[\tilde{\rho}_{s}^{i}\right]\right)-\mathbb{E}\left[f^{P}\left(\tilde{\rho}_{s-\delta}^{i}-\mathbb{E}\left[\tilde{\rho}_{s-\delta}^{i}\right]\right)\left(\tilde{\rho}_{s}^{i}-\mathbb{E}\left[\tilde{\rho}_{s}^{i}\right]\right)\right] \mid\right] d s=0 .\right.
$$

Since $\tilde{\rho}^{i}, i=1, \ldots, n$, are independent and identically distributed, we have that, for $\delta \leq s \leq$ $t$,

$$
\lim _{n \rightarrow \infty} \mathbb{E}\left[\left|\frac{1}{n} \sum_{i=1}^{n} f^{P}\left(\tilde{\rho}_{s-\delta}^{i}-\mathbb{E}\left[\tilde{\rho}_{s-\delta}^{i}\right]\right)\left(\tilde{\rho}_{s}^{i}-\mathbb{E}\left[\tilde{\rho}_{s}^{i}\right]\right)-\mathbb{E}\left[f^{P}\left(\tilde{\rho}_{s-\delta}^{i}-\mathbb{E}\left[\tilde{\rho}_{s-\delta}^{i}\right]\right)\left(\tilde{\rho}_{s}^{i}-\mathbb{E}\left[\tilde{\rho}_{s}^{i}\right]\right)\right]\right|\right]=0 .
$$

Then limit 3.20 follows by the dominated convergence theorem, by Assumption 2.1 and since the Ornstein-Uhlenbeck process has finite moments, see the computations in (3.18).

\section{Liquidity induced bubbles: theory and numerics}

\subsection{Liquidity induced bubbles in an information network}

We now provide more details on the theory of asset price bubbles and our model choice for $\beta$ in this paper. 
The formation of asset price bubbles has been thoroughly investigated from an economical point of view, see e.g. Tirole [71], Allen and Gale [2], Choi and Douady [20, 21], Harrison and Kreps [42], Kaizoji [53], Earl et al. [30], DeLong et al. [25], Scheinkman and Xiong [68, 69], Xiong [72, Abreu and Brunnermeier [1], Föllmer [35], Miller [63], Zhuk [73].

Different causes have been indicated as triggering factors for bubble birth, such as heterogenous beliefs between interacting agents (as in [35, [42, 66], 69, [72, [73]), a breakdown of the dynamic stability of the financial system ([21], [20]), the diffusion of new investment decision rules from a few expert investors to larger population of amateurs (see [30]), the tendency of traders to choose the same behaviour as the other traders' behaviour as thoroughly as possible (see [53]), the presence of short-selling constraints (see [63]).

From the mathematical point of view, financial asset bubbles have been mainly studied via the martingale theory of bubbles, introduced by Cox and Hobson [24] and Loewenstein and Willard [57] and mainly developed by Jarrow and Protter [47, 48, Jarrow et al. [49, 50, 51, Protter [67]. In this setting a $Q$-bubble is defined as the difference between the market price of a given financial asset and its fundamental value, given by the expectation of the future cash flows under an equivalent local martingale measure $Q$.

Furthermore, other constructive approaches have been proposed, where the fundamental value is exogenously given, whereas the market value is endogenously determined, see Jarrow et al. [52] and Biagini et al. [10].

We here follow the approach of [10] and [52] and assume that the market wealth is determined by the trading activity of investors and studied through the analysis of the liquidity supply curve. In particular, the stock is traded through a limit order book, so that limit orders and market orders are possible. Market orders, which deplete or fill in the limit order book, produce a variation in the price over a small interval of time. If new market orders quickly enter before the price has time to decay again to the fundamental value, these short-term price variations may accumulate and result in a deviation from the fundamental wealth with a consequent bubble birth ${ }^{2}$.

Motivated by the above analysis, the bubble is supposed to follow the dynamics

$$
d \beta_{t}=M_{t} \Lambda_{t}\left(-k \beta_{t} d t+2 d X_{t}\right), \quad t \geq 0
$$

where $M=\left(M_{t}\right)_{t \geq 0}$ and $\Lambda=\left(\Lambda_{t}\right)_{t \geq 0}$ are respectively a measure of illiquidity and the so called resiliency of the limit order book, which takes values in $[0,1]$. The process $X=\left(X_{t}\right)_{t \geq 0}$ is the signed volume of market orders, defined as the cumulated difference between the buy market orders and the sell market orders. Moreover, in agreement with the approach of [52], $k>0$ is the speed of decay, which is assumed to be strictly positive since the market price is supposed to go back to the fundamental value in the long term.

\footnotetext{
${ }^{2}$ For more details about the economical motivation of this setting, we refer to [10] and [52].
} 
We consider that $X$ satisfies the following dynamics

$$
d X_{t}=\bar{\mu}_{t} d t+\bar{\sigma}_{t} d B_{t}^{2}, \quad t \geq 0
$$

where $\bar{\mu}=\left(\bar{\mu}_{t}\right)_{t \geq 0}$ and $\bar{\sigma}=\left(\bar{\sigma}_{t}\right)_{t \geq 0}$ are progressively measurable processes satisfying some integrability conditions. In this way,

$$
d \beta_{t}=\Lambda_{t} M_{t}\left[\left(-k \beta_{t}+2 \bar{\mu}_{t}\right) d t+2 \bar{\sigma}_{t} d B_{t}^{2}\right], \quad t \geq 0
$$

i.e. $\beta$ solves 2.4 with

$$
\mu_{t}=M_{t} \Lambda_{t}\left(-k \beta_{t}+2 \bar{\mu}_{t}\right), \quad \sigma_{t}^{B}=2 \bar{\sigma}_{t} M_{t} \Lambda_{t}, \quad t \geq 0 .
$$

In the simulations below, the illiquidity $M$ is supposed to be a geometric Brownian motion, whereas $\Lambda$ is taken constant.

In [10], the evolution of $X$ is modelled through a contagion process within an information network of investors. Traders may imitate neighbours in the network that have successfully bought the bubbly asset, and place as a consequence a buy market order on the asset. This eventually leads to some self-exciting herding effect, which in turn blows up the signed volume of market orders and then generates the bubble. The analysis of the contagion mechanism is based on some epidemiological studies describing virus diffusion in a population. In particular, virus diffusion is reinterpreted as trading contagion, and modelled through the SIS model, studied for example by Pastor-Satorras and Vespignani [64, 65].

The evolution of the bubble is then characterised by two different phases: in the first one the bubble blows up, since the quick increase of the signed volume of market orders $X$ dominates in equation (4.1). In this phase, the essential force of the bubble is given by the contagion mechanism driving $X$. The contagion accelerates to a maximum and then slows down, since it tends towards an equilibrium. At this point, the drift of $X$ gets smaller, and the mean reverting term of equation (4.1) starts to dominate. This leads to the burst of the bubble, here identified by a stopping time time $\tau$, and to the second phase, i.e. the decrease of the bubble towards zero. In particular, in the next subsections we characterise $\tau$ as the first time when the drift in 4.3 gets negative.

\subsection{Risk analysis for the finite case}

We now study by numerical simulations how the system described in Section 3 reacts to the growth and the burst of a bubble. In particular, we investigate how a bank not holding the bubbly asset can be affected by a bubble burst through contagion mechanisms. We first consider the case of 2.1 $-(2.2)$, i.e. of a network with a finite number of banks, and then we analyse the limit system $(3.1)-(3.3)$. 
We choose the same function $f$ for both core and periphery banks in 2.1$)-(2.2)$, i.e. $f^{B}=$ $f^{P}=f$. In particular, we take $f(x)=1+2 \arctan (x) / \pi$, as in Example 2.2. We investigate how the first bank reacts when banks holding the bubble are in trouble. Specifically, we here introduce and compute the risk measure

$$
\operatorname{Risk}_{\alpha}^{i, \Delta}=-\sup \left\{x \in \mathbb{R}:\left[\frac{1}{N_{s}} \sum_{k=1}^{N_{s}} \mathbb{1}_{\left\{\rho_{\tau_{k}+\Delta}^{i, n, k}-\rho_{\tau_{k}}^{i, n, k} \leq x\right\}}\right] \leq \alpha\right\},
$$

with $\alpha>0$, where $N_{s}$ is the number of simulations of the processes in $(2.1)-(2.2), \tau_{k}$ is the value at the $k$-th simulation of the bursting time $\tau$ of the bubble, and $\rho_{t}^{i, n, k}$ is the value of $\rho_{t}^{i, n}$ computed in the $k$-th simulation. Here $\Delta$ represents a time interval after bubble burst, which can be considered as an exogenously given risk management time horizon.

The risk measure $\operatorname{Risk}_{\alpha}^{i, \Delta}$ as defined in (4.4) measures the impact of realised distress of the institutions holding the bubble on the system, at the moment of the burst. In this sense, it can be seen as the CoVar of a bank without the bubbly asset when banks holding the bubbly asset suffer a loss (for a definition of CoVar see e.g. Biagini et al. [8] and Brunnermeier and Oehmke [15]). Note that, since the banks not holding the bubble are identically distributed, we only compute the risk for one bank.

From now on, we set $\alpha=0.05$ in 4.4. We perform $N_{s}=10000$ simulations of Risk $k_{0.05}^{1, \Delta}$ in the case when there are $n=6$ banks not holding the bubble and $m=2$ banks holding it. We consider different values of $\lambda$ and of the delay $\delta$.

The results are given in Tables 1,2 and 3 for $\Delta=0.05,0.1,0.2$ respectively.

\begin{tabular}{|c|c|c|c|c|c|c|c|}
\hline & $\delta=0$ & $\delta=0.025$ & $\delta=0.05$ & $\delta=0.075$ & $\delta=0.1$ & $\delta=0.2$ & $\delta=0.3$ \\
\hline$\lambda=0.5$ & 0.109 & 0.150 & 0.292 & 0.289 & 0.290 & 0.288 & 0.286 \\
\hline$\lambda=1$ & 0.083 & 0.135 & 0.252 & 0.251 & 0.245 & 0.245 & 0.249 \\
\hline$\lambda=0.5$ & 0.083 & 0.119 & 0.230 & 0.227 & 0.226 & 0.225 & 0.222 \\
\hline
\end{tabular}

Table 1: Risk $k_{0.05}^{1, \Delta}$ in the case when the robustness is given by 2.1)-2.2), with parameters $\sigma_{1}=\sigma_{2}=0.2, \Delta=0.05, \rho_{0}^{i, 6}=\rho_{0}^{k, B}=0.5, i=1, \ldots, 6, k=1,2$.

\begin{tabular}{|c|c|c|c|c|c|c|c|}
\hline & $\delta=0$ & $\delta=0.025$ & $\delta=0.05$ & $\delta=0.075$ & $\delta=0.1$ & $\delta=0.2$ & $\delta=0.3$ \\
\hline$\lambda=0.5$ & 0.125 & 0.176 & 0.441 & 0.672 & 0.886 & 0.864 & 0.863 \\
\hline$\lambda=1$ & 0.121 & 0.163 & 0.301 & 0.540 & 0.777 & 0.758 & 0.751 \\
\hline$\lambda=2$ & 0.120 & 0.130 & 0.246 & 0.485 & 0.703 & 0.686 & 0.684 \\
\hline
\end{tabular}

Table 2: $\operatorname{Risk}_{0.05}^{1, \Delta}$ in the case when the robustness is given by (2.1)-(2.2), with parameters $\sigma_{1}=\sigma_{2}=0.2, \Delta=0.1, \rho_{0}^{i, 6}=\rho_{0}^{k, B}=0.5, i=1, \ldots, 6, k=1,2$. 


\begin{tabular}{|c|c|c|c|c|c|c|c|}
\hline & $\delta=0$ & $\delta=0.025$ & $\delta=0.05$ & $\delta=0.075$ & $\delta=0.1$ & $\delta=0.2$ & $\delta=0.3$ \\
\hline$\lambda=0.5$ & 0.261 & 0.294 & 0.493 & 0.753 & 1.085 & 2.070 & 2.007 \\
\hline$\lambda=1$ & 0.190 & 0.215 & 0.382 & 0.585 & 0.893 & 1.900 & 1.879 \\
\hline$\lambda=2$ & 0.170 & 0.181 & 0.330 & 0.535 & 0.785 & 1.812 & 1.806 \\
\hline
\end{tabular}

Table 3: $\operatorname{Risk}_{0.05}^{1, \Delta}$ in the case when the robustness is given by (2.1)-(2.2), with parameters $\sigma_{1}=\sigma_{2}=0.2, \Delta=0.2, \rho_{0}^{i, 6}=\rho_{0}^{k, B}=0.5, i=1, \ldots, 6, k=1,2$.

We note a non-monotonic behaviour with respect to the delay $\delta$ : when the delay is small, banks are able to quickly disinvest when other institutions holding the bubble are in trouble, reducing the loss. However, in all the three cases $\Delta=0.05, \Delta=0.1$ and $\Delta=0.2$, we observe that for delays larger than $\Delta$, the risk is still big but it decreases. This depends on the fact that we check the robustness of the banks at time $\tau+\Delta$ : at this time, when $\delta>\Delta, f$ is smaller than in the case $\delta=\Delta$ because banks are cross investing on each other according to a value of the robustness, which is realised much before the bubble's burst.

Moreover, the risk is decreasing with $\lambda$. Indeed, it follows by (2.1) that $\rho^{i, n}$ reverts to

$$
A_{t}^{n, m}+\frac{1}{\lambda}\left(\frac{1}{n} \sum_{i=1}^{n} f\left(\rho_{t-\delta}^{i, n}-A_{t-\delta}^{n, m}\right)\left(\rho_{t}^{i, n}-A_{t}^{n, m}\right)+\frac{1}{m-1} \sum_{\ell=1, \ell \neq k}^{m} f\left(\rho_{t-\delta}^{\ell, B}-A_{t-\delta}^{n, m}\right)\left(\rho_{t}^{\ell, n}-A_{t}^{n, m}\right)\right)
$$

so that for large $\lambda$ the term involving the network, and then the direct effects of the banks holding the bubbly asset, is less significative.

Remark 4. By (4.1), we note that the mean reversion term $-k \beta_{t}$ is the main driving force of the shock at the moment of the bubble's burst. This term dominates when the contagion mechanism triggering the bubble slows down. Since it is a linear function of the bubble, we see that the size of the bubble at the moment of the burst affects the risk in two ways:

- it amplifies the shock suffered by the banks holding the bubbly asset, through the above mentioned term $-k \beta_{t}$;

- it makes the network more centralised towards the banks detaining the bubbly asset. This is due to the fact that bubble's size also influences the term $f\left(\rho_{\tau-\delta}^{k, B}-A_{\tau-\delta}^{n, m}\right)$ in (2.1), so that banks in the periphery have a strong connection with the banks that suffer the shock. This makes the system more prone to systemic risk.

In order to investigate this last phenomenon, we now consider $(2.1)-(2.2)$ when $\beta$ is replaced by $\bar{\beta}$, where

$$
\bar{\beta}_{t}=\left\{\begin{array}{l}
0 \quad \text { for } t \leq \tau \\
\beta_{t}-\beta_{\tau} \quad \text { for } t>\tau
\end{array}\right.
$$


In this way we model the case when the banks that hold the bubbly asset are subject at time $\tau$ to the same shock, but without having experienced the growth of the bubble.

Remark 5. Note that we assume the same shock size in the scenario with and without the bubble. This is a conservative assumption, as the shock size would be expected to be smaller when there is no bubble. Considering the same shock in both scenarios allows to isolate the impact on systemic risk due to the distortion of the network's shape caused by the bubble. We see that even under this conservative assumption, the risk is smaller when there are no banks holding the bubbly asset.

The results are given in Tables 4 , 5 and 6 for $\Delta=0.05,0.1,0.2$ respectively.

\begin{tabular}{|c|c|c|c|c|c|c|c|}
\hline & $\delta=0$ & $\delta=0.025$ & $\delta=0.05$ & $\delta=0.075$ & $\delta=0.1$ & $\delta=0.2$ & $\delta=0.3$ \\
\hline$\lambda=0.5$ & 0.113 & 0.143 & 0.279 & 0.277 & 0.276 & 0.276 & 0.274 \\
\hline$\lambda=1$ & 0.085 & 0.130 & 0.224 & 0.222 & 0.221 & 0.220 & 0.217 \\
\hline$\lambda=2$ & 0.079 & 0.111 & 0.202 & 0.196 & 0.191 & 0.190 & 0.190 \\
\hline
\end{tabular}

Table 4: $\operatorname{Risk}_{0.05}^{1, \Delta}$ in the case when the robustness is given by 2.1)-2.2 with no bubble in the system, but with the same shock at time $\tau$, for parameters $\sigma_{1}=\sigma_{2}=0.2, \Delta=0.05$, $\rho_{0}^{i, 6}=\rho_{0}^{k, B}=0.5, i=1, \ldots, 6, k=1,2$.

\begin{tabular}{|c|c|c|c|c|c|c|c|}
\hline & $\delta=0$ & $\delta=0.025$ & $\delta=0.05$ & $\delta=0.075$ & $\delta=0.1$ & $\delta=0.2$ & $\delta=0.3$ \\
\hline$\lambda=0.5$ & 0.138 & 0.172 & 0.320 & 0.500 & 0.773 & 0.765 & 0.760 \\
\hline$\lambda=1$ & 0.122 & 0.160 & 0.259 & 0.434 & 0.641 & 0.611 & 0.600 \\
\hline$\lambda=2$ & 0.127 & 0.126 & 0.193 & 0.343 & 0.524 & 0.511 & 0.506 \\
\hline
\end{tabular}

Table 5: $\operatorname{Risk}_{0.05}^{1, \Delta}$ in the case when the robustness is given by (2.1)-2.2 with no bubble in the system, but with the same shock at time $\tau$, for parameters $\sigma_{1}=\sigma_{2}=0.2, \Delta=0.1$, $\rho_{0}^{i, 6}=\rho_{0}^{k, B}=0.5, i=1, \ldots, 6, k=1,2$.

\begin{tabular}{|c|c|c|c|c|c|c|c|}
\hline & $\delta=0$ & $\delta=0.025$ & $\delta=0.05$ & $\delta=0.075$ & $\delta=0.1$ & $\delta=0.2$ & $\delta=0.3$ \\
\hline$\lambda=0.5$ & 0.268 & 0.290 & 0.425 & 0.680 & 0.999 & 1.773 & 1.765 \\
\hline$\lambda=1$ & 0.187 & 0.210 & 0.356 & 0.480 & 0.695 & 1.449 & 1.412 \\
\hline$\lambda=2$ & 0.173 & 0.180 & 0.202 & 0.447 & 0.570 & 1.269 & 1.262 \\
\hline
\end{tabular}

Table 6: $R i s k_{0.05}^{1, \Delta}$ in the case when the robustness is given by (2.1)-2.2) with no bubble in the system, but with the same shock at time $\tau$, for parameters $\sigma_{1}=\sigma_{2}=0.2, \Delta=0.2$, $\rho_{0}^{i, 6}=\rho_{0}^{k, B}=0.5, i=1, \ldots, 6, k=1,2$. 
Remark 6. We note that, also in this case, the risk is maximum when $\delta=\Delta$. This means that the risk first increases and then (slightly) decreases with respect to the delay not because of the presence of the bubble, but due to the nature of the system (2.1)-(2.2). Of course when the delay is small, the risk is also smaller, because banks can promptly disinvest when the others are hit by the shock. However, the behaviour for delays larger than $\Delta$ is more subtle. Even if there is no bubble, the robustness of some banks in the system may be bigger than the rest, because of the random effect of Brownian motions. In the case under examination, the worst scenarios occur when $\zeta_{t, \delta}:=\rho_{t-\delta}^{k, B}-A_{t-\delta}^{n, m}$ is big for $t \in[\tau, \tau+\Delta]$, so that the banks have a stronger link towards the ones hit by the shock. This happens for the choice $\alpha=0.05$ in (4.4), if $\delta \geq \Delta$. Moreover, $\zeta_{t, \delta}$ is slightly smaller for large delays, if $t-\delta \leq \tau$ (which is the case for every $t \in[\tau, \tau+\Delta]$ if $\delta \geq \Delta)$. For this reason, Risk $k_{0.05}^{1, \Delta}$ is smaller when $\delta>\Delta$ compared to the case $\delta=\Delta$.

We now compare the results to the case when there is a bubble in the system. Note that for $\delta=0$ there is not any significant difference, since the banks are able to disinvest immediately at the time when the shock hits the banks with the bubble. Anyway, this difference increases with the delay. When the delay is big, the banks with no bubble are much more in trouble in the first case, i.e when they are attached to banks holding the bubbly asset.

We can then conclude that the increase of the value of the bubbly asset can put the network in trouble, because it makes the system more centralised on the riskier banks, due to the preferential attachment mechanism implied by (2.1)- 2.2 .

This can also be seen by considering a static network, i.e. by taking $f^{B}=f^{P}=1$ in (2.1)- (2.2), see Table 7.

\begin{tabular}{|c|c|c|c|}
\hline & $\Delta=0.05$ & $\Delta=0.1$ & $\Delta=0.2$ \\
\hline$\lambda=0.5$ & 0.251 & 0.702 & 1.821 \\
\hline$\lambda=1$ & 0.191 & 0.533 & 1.754 \\
\hline$\lambda=2$ & 0.158 & 0.437 & 1.765 \\
\hline
\end{tabular}

Table 7: Risk $k_{0.05}^{1, \Delta}$ in the case of a static network, with $f^{B}=f^{P}=1$ and with parameters $\sigma_{1}=\sigma_{2}=0.2, \rho_{0}^{i, 6}=\rho_{0}^{k, B}=0.5, i=1, \ldots, 6, k=1,2$.

Note that in this case the delay plays no role since it only affects the dynamics through $f^{B}$ and $f^{P}$. Comparing this result with Tables 1,2 and 3 , we see that, when $\delta<\Delta$, the fact that banks are able to disinvest before the risk management time horizon $\Delta$ makes the measure Risk $_{0.05}^{1, \Delta}$ smaller than in the case of a static network. On the other hand, for big values of $\delta$, a centralised network towards the banks holding the bubbly asset and the impossibility to quickly disinvest after the burst give rise to a more dangerous system than in the static case. 


\subsection{Risk analysis for the mean field limit}

We now consider the case of the limit system (3.1)-(3.3). We compute

$$
\operatorname{Risk}_{0.05}^{1, \Delta}=-\sup \left\{x \in \mathbb{R}:\left[\frac{1}{N_{s}} \sum_{k=1}^{N_{s}} \mathbb{1}_{\left\{\bar{\rho}_{\tau_{k}+\Delta}^{1, k}-\bar{\rho}_{\tau_{k}}^{1, k} \leq x\right\}}\right] \leq 0.05\right\},
$$

where $N_{s}$ and $\tau_{k}$ are the number of simulations and the time of the burst of the bubble in the $k$-th simulation, respectively, and $\bar{\rho}_{t}^{1, k}$ is the value of $\bar{\rho}_{t}^{1}$ computed in the $k$-th simulation.

As before, we consider $m=2$ banks holding the bubble and we make $N_{s}=10000$ simulations of (3.1)-3.3 taking different values of $\lambda, \delta$ and $\Delta$.

Note that, calling $\mu_{\tilde{\rho}, s}=\rho_{0} e^{-\lambda s}$ and $\sigma_{\tilde{\rho}, s}=\frac{\left(\sigma_{1}\right)^{2}}{2 \theta}\left(1-e^{-2 \theta s}\right)$ the expectation and the variance of $\tilde{\rho}_{s}^{i}$, we can directly compute $\varphi(t, t-\delta)$ in $(3.4)$ with $f^{p}(x)=1+\frac{2}{\pi} \arctan (x)$ as

$$
\begin{aligned}
\varphi(t, t-\delta)= & e^{-\lambda \delta}\left[f^{P}\left(\tilde{\rho}_{t-\delta}^{i}-\mathbb{E}\left[\tilde{\rho}_{t-\delta}^{i}\right]\right) \tilde{\rho}_{t-\delta}^{i}\right]-\mu_{\tilde{\rho}, t} \mathbb{E}\left[f^{P}\left(\tilde{\rho}_{t-\delta}^{i}-\mathbb{E}\left[\tilde{\rho}_{t-\delta}^{i}\right]\right)\right] \\
= & e^{-\lambda \delta} \int_{-\infty}^{\infty} \frac{1}{\sqrt{2 \sigma_{\tilde{\rho}, t-\delta} \pi}} e^{-\left(x-\mu_{\tilde{\rho}, t-\delta}\right)^{2} /\left(2 \sigma_{\tilde{\rho}, t-\delta}\right)}\left(1+\frac{2}{\pi} \arctan \left(x-\mu_{\tilde{\rho}, t-\delta}\right)\right) x d x \\
& -\mu_{\tilde{\rho}, t} \int_{-\infty}^{\infty} \frac{1}{\sqrt{2 \sigma_{\tilde{\rho}, t-\delta} \pi}} e^{-\left(x-\mu_{\tilde{\rho}, t-\delta}\right)^{2} /\left(2 \sigma_{\tilde{\rho}, t-\delta}\right)}\left(1+\frac{2}{\pi} \arctan \left(x-\mu_{\tilde{\rho}, t-\delta}\right)\right) d x \\
= & e^{-\lambda \delta} \frac{2}{\pi} \int_{-\infty}^{\infty} \frac{1}{\sqrt{2 \sigma_{\tilde{\rho}, t-\delta} \pi}} e^{-\left(x-\mu_{\tilde{\rho}, t-\delta}\right)^{2} /\left(2 \sigma_{\tilde{\rho}, t-\delta}\right)} \arctan \left(x-\mu_{\tilde{\rho}, t-\delta}\right)\left(x-\mu_{\tilde{\rho}, t-\delta}\right) d x \\
& +\frac{2}{\pi}\left(e^{-\lambda \delta}-1\right) \mu_{\tilde{\rho}, t-\delta} \int_{-\infty}^{\infty} \frac{1}{\sqrt{2 \sigma_{\tilde{\rho}, t-\delta} \pi}} e^{-\left(x-\mu_{\tilde{\rho}, t-\delta}\right)^{2} /\left(2 \sigma_{\tilde{\rho}, t-\delta}\right)} \arctan \left(x-\mu_{\tilde{\rho}, t-\delta}\right) d x \\
= & e^{-\lambda \delta} \frac{2}{\pi} \int_{-\infty}^{\infty} \frac{1}{\sqrt{2 \sigma_{\tilde{\rho}, t-\delta} \pi}} e^{-x^{2} /\left(2 \sigma_{\tilde{\rho}, t-\delta}\right)} \arctan (x) x d x \\
= & e^{-\lambda \delta+1 /\left(2 \sigma_{\tilde{\rho}, t-\delta}\right)} \sqrt{\sigma_{\tilde{\rho}, t-\delta}} \sqrt{\frac{2}{\pi}} \operatorname{Erfc}\left(1 / \sqrt{2 \sigma_{\tilde{\rho}, t-\delta}}\right), \quad 0 \leq \delta \leq t,
\end{aligned}
$$

with $\operatorname{Erfc}(x)=\frac{2}{\sqrt{\pi}} \int_{x}^{\infty} e^{-t^{2}} d t$, where we have used the fact that $\int_{-\infty}^{\infty} e^{-x^{2} /\left(2 \sigma_{\tilde{\rho}, t-\delta}^{2}\right)} \arctan (x) d x=$ 0 .

The results of the simulations are gathered in Tables 8 , 9 and 10 for $\Delta=0.05,0.1,0.2$, respectively.

\begin{tabular}{|c|c|c|c|c|c|c|c|}
\hline & $\delta=0$ & $\delta=0.025$ & $\delta=0.05$ & $\delta=0.075$ & $\delta=0.1$ & $\delta=0.2$ & $\delta=0.3$ \\
\hline$\lambda=0.5$ & 0.078 & 0.169 & 0.331 & 0.320 & 0.318 & 0.315 & 0.313 \\
\hline$\lambda=1$ & 0.087 & 0.168 & 0.327 & 0.315 & 0.311 & 0.311 & 0.308 \\
\hline$\lambda=2$ & 0.085 & 0.160 & 0.325 & 0.313 & 0.309 & 0.307 & 0.302 \\
\hline
\end{tabular}

Table 8: Risk $k_{0.05}^{1, \Delta}$ with $\Delta=0.05$ of the mean field limit $3.1-(3.3)$, with parameters $\sigma_{1}=$ $\sigma_{2}=0.2, \rho_{0}^{k, B}=0.5, k=1,2$. 


\begin{tabular}{|c|c|c|c|c|c|c|c|}
\hline & $\delta=0$ & $\delta=0.025$ & $\delta=0.05$ & $\delta=0.075$ & $\delta=0.1$ & $\delta=0.2$ & $\delta=0.3$ \\
\hline$\lambda=0.5$ & 0.134 & 0.210 & 0.442 & 0.762 & 1.043 & 1.041 & 1.040 \\
\hline$\lambda=1$ & 0.131 & 0.215 & 0.428 & 0.739 & 1.015 & 1.011 & 1.010 \\
\hline$\lambda=2$ & 0.128 & 0.213 & 0.425 & 0.663 & 0.918 & 0.909 & 0.909 \\
\hline
\end{tabular}

Table 9: Risk $k_{0.05}^{1, \Delta}$ with $\Delta=0.1$ of the mean field limit 3.1 - -3.3 , with parameters $\sigma_{1}=$ $\sigma_{2}=0.2, \rho_{0}^{k, B}=0.5, k=1,2$.

\begin{tabular}{|c|c|c|c|c|c|c|c|}
\hline & $\delta=0$ & $\delta=0.025$ & $\delta=0.05$ & $\delta=0.075$ & $\delta=0.1$ & $\delta=0.2$ & $\delta=0.3$ \\
\hline$\lambda=0.5$ & 0.218 & 0.297 & 0.512 & 0.827 & 1.192 & 2.764 & 2.710 \\
\hline$\lambda=1$ & 0.215 & 0.295 & 0.510 & 0.815 & 1.152 & 2.586 & 2.547 \\
\hline$\lambda=2$ & 0.215 & 0.286 & 0.488 & 0.736 & 1.027 & 2.377 & 2.307 \\
\hline
\end{tabular}

Table 10: $\operatorname{Risk}_{0.05}^{1, \Delta}$ with $\Delta=0.2$ of the mean field limit (3.1)-(3.3), with parameters $\sigma_{1}=$ $\sigma_{2}=0.2, \rho_{0}^{k, B}=0.5, k=1,2$.

As before, the risk is increasing with the delay until $\delta=\Delta$ and decreasing with $\lambda$, since $\bar{\rho}_{t}^{i}$ reverts to

$$
\frac{1}{\lambda}\left(\varphi(t, t-\delta)+\frac{1}{m} \sum_{k=1}^{m} f\left(\bar{\rho}_{t-\delta}^{k, B}-\nu_{t-\delta}-\mathbb{E}\left[\tilde{\rho}_{t-\delta}^{i}\right]\right)\left(\bar{\rho}_{t}^{k, B}-\nu_{t}-\mathbb{E}\left[\tilde{\rho}_{t}^{i}\right]\right)\right)+\mathbb{E}\left[\tilde{\rho}_{t}^{i}\right]-\tilde{\rho}_{t}^{i}
$$

so that a large $\lambda$ diminishes the influence of the banks holding the bubbly asset.

We can also see that the risk is bigger at the limit by comparing (2.1) and (3.6): since $\nu_{t-\delta}+\mathbb{E}\left[\tilde{\rho}_{t}^{i}\right]<A_{t-\delta}^{n, m}$, because the first term is the average robustness of banks not holding the bubble, the argument of $f$ is bigger in (3.6). This leads to a bigger weight multiplying the loss at the moment of the burst at the limit.

In Tables 11, 12 and 13 we report the results for the case when $\beta$ is replaced by $\bar{\beta}$ as in $(4.5)$, i.e. when there is no bubble in the network, and $\Delta=0.05,0.1,0.2$, respectively.

\begin{tabular}{|c|c|c|c|c|c|c|c|}
\hline & $\delta=0$ & $\delta=0.025$ & $\delta=0.05$ & $\delta=0.075$ & $\delta=0.1$ & $\delta=0.2$ & $\delta=0.3$ \\
\hline$\lambda=0.5$ & 0.087 & 0.152 & 0.281 & 0.279 & 0.274 & 0.274 & 0.269 \\
\hline$\lambda=1$ & 0.091 & 0.152 & 0.280 & 0.277 & 0.274 & 0.273 & 0.268 \\
\hline$\lambda=2$ & 0.088 & 0.151 & 0.275 & 0.272 & 0.270 & 0.267 & 0.264 \\
\hline
\end{tabular}

Table 11: Risk $k_{0.05}^{1, \Delta}$ with $\Delta=0.05$ of the mean field limit (3.1)-(3.3) with no bubble in the system, but with the same shock at time $\tau$, with parameters $\sigma_{1}=\sigma_{2}=0.2, \rho_{0}^{k, B}=0.5$, $k=1,2$. 


\begin{tabular}{|c|c|c|c|c|c|c|c|}
\hline & $\delta=0$ & $\delta=0.025$ & $\delta=0.05$ & $\delta=0.075$ & $\delta=0.1$ & $\delta=0.2$ & $\delta=0.3$ \\
\hline$\lambda=0.5$ & 0.142 & 0.200 & 0.369 & 0.583 & 0.801 & 0.799 & 0.791 \\
\hline$\lambda=1$ & 0.137 & 0.205 & 0.359 & 0.579 & 0.790 & 0.788 & 0.770 \\
\hline$\lambda=2$ & 0.140 & 0.203 & 0.357 & 0.559 & 0.759 & 0.753 & 0.740 \\
\hline
\end{tabular}

Table 12: Risk $k_{0.05}^{1, \Delta}$ with $\Delta=0.1$ of the mean field limit (3.1)-(3.3) with no bubble in the system, but with the same shock at time $\tau$, with parameters $\sigma_{1}=\sigma_{2}=0.2, \rho_{0}^{k, B}=0.5$, $k=1,2$.

\begin{tabular}{|c|c|c|c|c|c|c|c|}
\hline & $\delta=0$ & $\delta=0.025$ & $\delta=0.05$ & $\delta=0.075$ & $\delta=0.1$ & $\delta=0.2$ & $\delta=0.3$ \\
\hline$\lambda=0.5$ & 0.229 & 0.294 & 0.445 & 0.670 & 0.919 & 2.011 & 1.976 \\
\hline$\lambda=1$ & 0.219 & 0.285 & 0.443 & 0.655 & 0.897 & 1.882 & 1.858 \\
\hline$\lambda=2$ & 0.219 & 0.280 & 0.433 & 0.630 & 0.875 & 1.735 & 1.724 \\
\hline
\end{tabular}

Table 13: Risk $k_{0.05}^{1, \Delta}$ with $\Delta=0.2$ of the mean field limit (3.1)-(3.3) with no bubble in the system, but with the same shock at time $\tau$, with parameters $\sigma_{1}=\sigma_{2}=0.2, \rho_{0}^{k, B}=0.5$, $k=1,2$.

As before, it can be seen that, when the delay is large enough, the preferential attachment mechanism, that takes place during the ascending phase of the bubble, creates a network more exposed to systemic risk at the time of the shock. This is made explicit by the term $f\left(\bar{\rho}_{t-\delta}^{k, B}-\nu_{t-\delta}-\mathbb{E}\left[\tilde{\rho}_{t-\delta}^{i}\right]\right)$ in $(3.2)$, which is big in the presence of a bubble, see also Remark 3. If we consider a a static network, with $f^{B}=f^{P}=1$, the results, shown in Table 14 . agree with the ones obtained in the case of the finite network: for small delays the dynamic network is less exposed to systemic risk with respect to the static one, whereas when the delay increases and the banks in the dynamic network are slower in disinvesting, the risk is bigger than for the static network.

\begin{tabular}{|c|c|c|c|}
\hline & $\Delta=0.05$ & $\Delta=0.1$ & $\Delta=0.2$ \\
\hline$\lambda=0.5$ & 0.256 & 0.690 & 1.754 \\
\hline$\lambda=1$ & 0.248 & 0.677 & 1.750 \\
\hline$\lambda=2$ & 0.235 & 0.675 & 1.681 \\
\hline
\end{tabular}

Table 14: Risk $k_{0.05}^{1, \Delta}$ in the case of a static network with $f^{B}=f^{P}=1$ of the mean field limit, with parameters $\sigma_{1}=\sigma_{2}=0.2, \rho_{0}^{k, B}=0.5, k=1,2$.

Remark 7. By comparing the tables of Section 4.2 and Section 4.3 , we see that the choice of the risk management time horizon $\Delta$ does not strongly impact the qualitative behaviour 
of the results: for every choice of $\Delta, \operatorname{Risk}_{0.05}^{1, \Delta}$ is bigger in the presence of the bubble, and is decreasing with respect to the parameter $\lambda$. For all values of $\Delta$, Risk $_{0.05}^{1, \Delta}$ is maximum when $\delta=\Delta$, see also Remark 6 .

In order to further display the effects of the bubble and of the delay, we present some graphics as well. Figures 1 and 2 show the evolution of a bank in periphery (for the same realisation of the driving Brownian motion, i.e. for the same $\omega \in \Omega$ ) in the case when the banks of the core own a bubbly asset and in the case when they suffer the same shock at the time of the burst, but without having experienced the growth of the bubble. The value of the robustness of the bank in the periphery at the time when the shock hits the banks in the core is marked with a black cross. We see that, immediately after the burst, the robustness of the bank continues to grow, because core banks' robustness is higher than the average in the term

$$
\frac{1}{m} \sum_{k=1}^{m} f^{B}\left(\bar{\rho}_{t-\delta}^{k, B}-\nu_{t-\delta}-\mathbb{E}\left[\tilde{\rho}_{t-\delta}^{i}\right]\right)\left(\bar{\rho}_{t}^{k, B}-\nu_{t}-\mathbb{E}\left[\tilde{\rho}_{t}^{i}\right]\right) .
$$

However, after a while, 4.8 gets negative and the bank is also indirectly impacted by the shock. The decrease of the robustness is higher in the case with the bubble, and for $\delta=\Delta$.
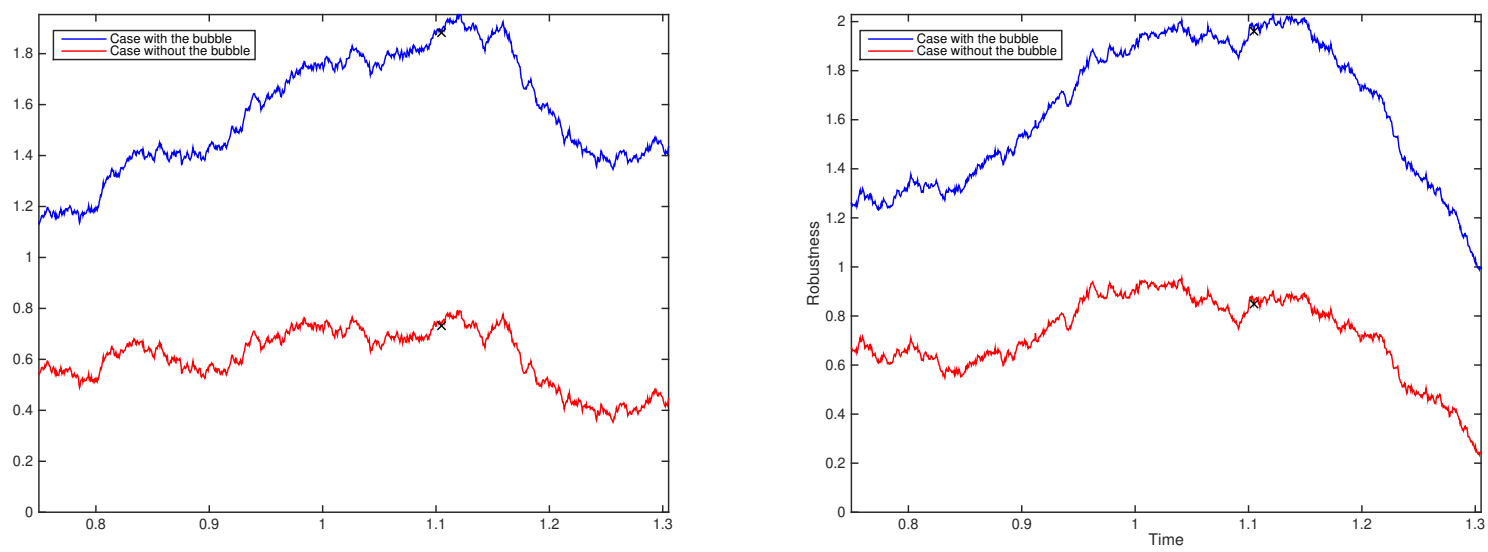

Figure 1: Evolution of the robustness of a Figure 2: Evolution of the robustness of a bank of the periphery in the limit system, with and without a bubble in the market, bank of the periphery in the limit system, with and without a bubble in the market, but with the same shock at time $\tau$, with parameters $\sigma_{1}=\sigma_{2}=0.2, \rho_{0}^{k, B}=0.5$, but with the same shock at time $\tau$, with $\delta=0.1$ and $\Delta=0.2$. parameters $\sigma_{1}=\sigma_{2}=0.2, \rho_{0}^{k, B}=0.5$, $\delta=0.2$ and $\Delta=0.2$.

The impact of the delay $\delta$ on the risk is further illustrated by Figure 3, where the robustness of a bank in the periphery is plotted for different values of $\delta$, again for the same 
$\omega \in \Omega$. Here we can see the behaviour described in Remark 6 ; when $\delta=0$ the bank can immediately disinvest when the banks in the core get in trouble, and thus its robustness does not decrease after the shock. However, when $\delta$ gets bigger, the decline of the robustness is more pronounced: for example, the decrease for $\delta=0.1$ and $\delta=0.2$ is the same up to $\tau+0.1$, but after $\tau+0.1$ the bank disinvest and stops the decrease if $\delta=0.1$, whereas it continues to $\operatorname{sink}$ if $\delta=0.2$.

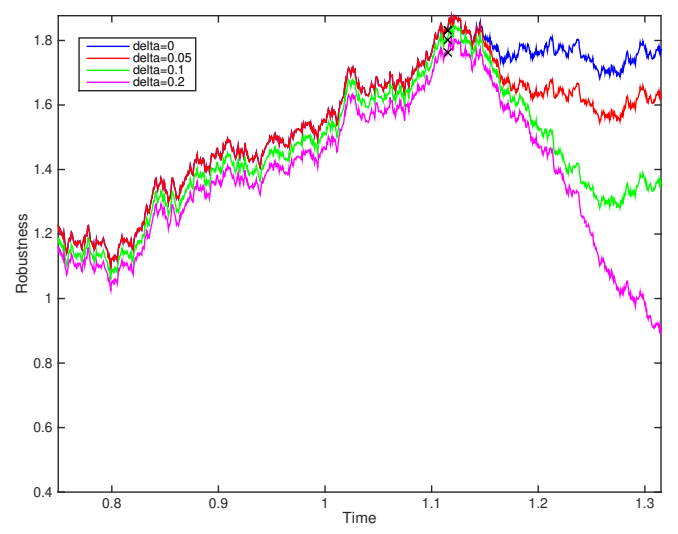

Figure 3: Evolution of the robustness of a bank of the periphery in the limit system, with parameters $\sigma_{1}=\sigma_{2}=0.2, \rho_{0}^{k, B}=0.5, \Delta=0.2$ and different values of the delay $\delta$.

\section{A Proof of Theorem 3.2}

We suppose by simplicity $\lambda=1$ and we proceed by steps, starting from the case when $0 \leq t<\delta$, i.e. when there is no delay in equations $(2.1)-(2.2)$ and $(3.2)-(3.3)$.

First step: case $0 \leq t<\delta$.

For every $i=1, \ldots, n$ and $t \in[0, \delta)$, we have

$$
\rho_{t}^{i, n}-\bar{\rho}_{t}^{i}=\int_{0}^{t} \Delta_{s}^{n} d s,
$$

where

$$
\begin{aligned}
\Delta_{s}^{n}= & \frac{1}{n-1} \sum_{j=1, j \neq i}^{n} f^{P}\left(\rho_{s}^{j, n}-A_{s}^{n, m}\right)\left(\rho_{s}^{j, n}-A_{s}^{n, m}\right)-\mathbb{E}\left[f^{P}\left(\tilde{\rho}_{s}^{i}-\mathbb{E}\left[\tilde{\rho}_{s}^{i}\right]\right)\left(\tilde{\rho}_{s}^{i}-\mathbb{E}\left[\tilde{\rho}_{s}^{i}\right]\right)\right] \\
& +\frac{1}{m} \sum_{k=1}^{m}\left(f^{B}\left(\rho_{s}^{k, B}-A_{s}^{n, m}\right)\left(\rho_{s}^{k, B}-A_{s}^{n, m}\right)-f^{B}\left(\bar{\rho}_{s}^{k, B}-\nu_{s}^{n}-\mathbb{E}\left[\tilde{\rho}_{s}^{i}\right]\right)\left(\bar{\rho}_{s}^{k, B}-\nu_{s}-\mathbb{E}\left[\tilde{\rho}_{s}^{i}\right]\right)\right) \\
& -\left(\rho_{s}^{i, n}-\bar{\rho}_{s}^{i}\right)+\left(A_{s}^{n, m}-\bar{A}_{s}^{n, m}\right)+\left(\bar{A}_{s}^{n, m}-\mathbb{E}\left[\tilde{\rho}_{s}^{i}\right]-\nu_{s}\right) .
\end{aligned}
$$


Thus

$$
\left|\rho^{i, n}-\bar{\rho}^{i}\right|_{t}^{*}=\sup _{s \leq t}\left|\int_{0}^{s} \Delta_{u}^{n} d u\right| \leq \sup _{s \leq t} \int_{0}^{s}\left|\Delta_{u}^{n}\right| d u=\int_{0}^{t}\left|\Delta_{u}^{n}\right| d u .
$$

Therefore, for every $i=1, \ldots, n$ and $t \geq 0$, we have

$$
\begin{aligned}
& \mathbb{E}\left[\left|\rho^{i, n}-\bar{\rho}^{i}\right|_{t}^{*}\right] \leq \mathbb{E}\left[\int_{0}^{t}\left|\Delta_{s}^{n}\right| d s\right] \\
& \leq \int_{0}^{t} \mathbb{E}\left[\left|\frac{1}{n-1} \sum_{j=1, j \neq i}^{n}\left(f^{P}\left(\rho_{s}^{j, n}-A_{s}^{n, m}\right)\left(\rho_{s}^{j, n}-A_{s}^{n, m}\right)-f^{P}\left(\bar{\rho}_{s}^{j}-\bar{A}_{s}^{n, m}\right)\left(\bar{\rho}_{s}^{j}-\bar{A}_{s}^{n, m}\right)\right)\right|\right] d s \\
& +\int_{0}^{t} \mathbb{E}\left[\left|\frac{1}{n-1} \sum_{j=1, j \neq i}^{n} f^{P}\left(\bar{\rho}_{s}^{j}-\bar{A}_{s}^{n, m}\right)\left(\bar{\rho}_{s}^{j}-\bar{A}_{s}^{n, m}\right)-\mathbb{E}\left[f^{P}\left(\tilde{\rho}_{s}^{i}-\mathbb{E}\left[\tilde{\rho}_{s}^{i}\right]\right)\left(\tilde{\rho}_{s}^{i}-\mathbb{E}\left[\tilde{\rho}_{s}^{i}\right]\right)\right]\right|\right] d s \\
& +\int_{0}^{t} \mathbb{E}\left[\left|\frac{1}{m} \sum_{k=1}^{m}\left(f^{B}\left(\rho_{s}^{k, B}-A_{s}^{n, m}\right)\left(\rho_{s}^{k, B}-A_{s}^{n, m}\right)-f^{B}\left(\bar{\rho}_{s}^{k, B}-\bar{A}_{s}^{n, m}\right)\left(\bar{\rho}_{s}^{k, B}-\bar{A}_{s}^{n, m}\right)\right)\right|\right] d s \\
& +\int_{0}^{t} \mathbb{E}\left[\frac{1}{m} \sum_{k=1}^{m}\left|f^{B}\left(\bar{\rho}_{s}^{k, B}-\bar{A}_{s}^{n, m}\right)\left(\bar{\rho}_{s}^{k, B}-\bar{A}_{s}^{n, m}\right)-f^{B}\left(\bar{\rho}_{s}^{k, B}-\nu_{s}^{n}-\mathbb{E}\left[\tilde{\rho}_{s}^{i}\right]\right)\left(\bar{\rho}_{s}^{k, B}-\nu_{s}-\mathbb{E}\left[\tilde{\rho}_{s}^{i}\right]\right)\right|\right] d s \\
& \quad+\int_{0}^{t} \mathbb{E}\left[\left|\rho_{s}^{i, n}-\bar{\rho}_{s}^{i}\right|\right] d s+\int_{0}^{t} \mathbb{E}\left[\left|A_{s}^{n, m}-\bar{A}_{s}^{n, m}\right|\right] d s+\int_{0}^{t} \mathbb{E}\left[\left|\bar{A}_{s}^{n, m}-\mathbb{E}\left[\tilde{\rho}_{s}^{i}\right]-\nu_{s}\right|\right] d s .
\end{aligned}
$$

By (2.9),

$$
\begin{aligned}
& \int_{0}^{t} \mathbb{E}\left[\left|\frac{1}{n-1} \sum_{j=1, j \neq i}^{n}\left(f^{P}\left(\rho_{s}^{j, n}-A_{s}^{n, m}\right)\left(\rho_{s}^{j, n}-A_{s}^{n, m}\right)-f^{P}\left(\bar{\rho}_{s}^{j}-\bar{A}_{s}^{n, m}\right)\left(\bar{\rho}_{s}^{j}-\bar{A}_{s}^{n, m}\right)\right)\right|\right] d s \\
& \quad \leq \frac{1}{n-1} \sum_{j=1, j \neq i}^{n} \int_{0}^{t} \mathbb{E}\left[\left|f^{P}\left(\rho_{s}^{j, n}-A_{s}^{n, m}\right)\left(\rho_{s}^{j, n}-A_{s}^{n, m}\right)-f^{P}\left(\bar{\rho}_{s}^{j}-\bar{A}_{s}^{n, m}\right)\left(\bar{\rho}_{s}^{j}-\bar{A}_{s}^{n, m}\right)\right|\right] d s \\
& \quad \leq K_{1} \frac{1}{n-1} \sum_{j=1, j \neq i}^{n} \int_{0}^{t} \mathbb{E}\left[\left|\left(\rho_{s}^{j, n}-A_{s}^{n, m}\right)-\left(\bar{\rho}_{s}^{j}-\bar{A}_{s}^{n, m}\right)\right|\right] d s \\
& \leq K_{1} \frac{1}{n-1} \sum_{j=1, j \neq i}^{n} \int_{0}^{t} \mathbb{E}\left[\left|\rho_{s}^{j, n}-\bar{\rho}_{s}^{j}\right|+\left|A_{s}^{n, m}-\bar{A}_{s}^{n, m}\right|\right] d s \\
& \quad=K_{1} \int_{0}^{t} \mathbb{E}\left[\left|\rho_{s}^{i, n}-\bar{\rho}_{s}^{i}\right|\right] d s+K_{1} \int_{0}^{t} \mathbb{E}\left[\left|A_{s}^{n, m}-\bar{A}_{s}^{n, m}\right|\right] d s, \quad t \geq 0 .
\end{aligned}
$$

By (2.3) and (3.15) we have that

$$
\int_{0}^{t} \mathbb{E}\left[\left|A_{s}^{n, m}-\bar{A}_{s}^{n, m}\right|\right] d s \leq \int_{0}^{t} \mathbb{E}\left[\frac{1}{m+n} \sum_{r=1}^{n}\left|\rho_{s}^{r, n}-\bar{\rho}_{s}^{r}\right|\right] d s+\int_{0}^{t} \mathbb{E}\left[\frac{1}{m+n} \sum_{k=1}^{m}\left|\rho_{s}^{h, B}-\bar{\rho}_{s}^{h, B}\right|\right] d s
$$


$\leq \int_{0}^{t} \mathbb{E}\left[\left|\rho_{s}^{i, n}-\bar{\rho}_{s}^{i}\right|\right] d s+\int_{0}^{t} \mathbb{E}\left[\left|\rho_{s}^{k, B}-\bar{\rho}_{s}^{k, B}\right|\right] d s, \quad t \geq 0$,

because all $\rho^{i}, i=1, \ldots, n$, and $\rho^{k, B}, k=1, \ldots, m$, are identically distributed.

We can conclude by (A.2) and A.3) that

$$
\begin{aligned}
& \int_{0}^{t} \mathbb{E}\left[\left|\frac{1}{n-1} \sum_{j=1, j \neq i}^{n}\left(f^{P}\left(\rho_{s}^{j, n}-A_{s}^{n, m}\right)\left(\rho_{s}^{j, n}-A_{s}^{n, m}\right)-f^{P}\left(\bar{\rho}_{s}^{j}-\bar{A}_{s}^{n, m}\right)\left(\bar{\rho}_{s}^{j}-\bar{A}_{s}^{n, m}\right)\right)\right|\right] d s \\
& \quad \leq 2 K_{1} \int_{0}^{t} \mathbb{E}\left[\left|\rho_{s}^{i, n}-\bar{\rho}_{s}^{i}\right|\right] d s+K_{1} \int_{0}^{t} \mathbb{E}\left[\left|\rho_{s}^{k, B}-\bar{\rho}_{s}^{k, B}\right|\right] d s \\
& \quad \leq 2 K_{1} \int_{0}^{t} \mathbb{E}\left[\left|\rho^{i, n}-\bar{\rho}^{i}\right|_{s}^{*}\right] d s+K_{1} \int_{0}^{t} \mathbb{E}\left[\left|\rho^{k, B}-\bar{\rho}^{k, B}\right|_{s}^{*}\right] d s, \quad t \geq 0 .
\end{aligned}
$$

Similarly,

$$
\begin{gathered}
\int_{0}^{t} \mathbb{E}\left[\left|\frac{1}{m} \sum_{k=1}^{m}\left(f^{B}\left(\rho_{s}^{k, B}-A_{s}^{n, m}\right)\left(\rho_{s}^{k, B}-A_{s}^{n, m}\right)-f^{B}\left(\bar{\rho}_{s}^{k, B}-\bar{A}_{s}^{n, m}\right)\left(\bar{\rho}_{s}^{k, B}-\bar{A}_{s}^{n, m}\right)\right)\right|\right] d s \\
\leq K_{1} \int_{0}^{t} \mathbb{E}\left[\left|\rho^{i, n}-\bar{\rho}^{i}\right|_{s}^{*}\right] d s+2 K_{1} \int_{0}^{t} \mathbb{E}\left[\left|\rho^{k, B}-\bar{\rho}^{k, B}\right|_{s}^{*}\right] d s \quad t \geq 0 .
\end{gathered}
$$

From A.1 , A.3 , A.4 and A.5 we have that

$$
\begin{aligned}
& \mathbb{E}\left[\left|\rho^{i, n}-\bar{\rho}^{i}\right|_{t}^{*}\right] \\
& \leq\left(3 K_{1}+2\right) \int_{0}^{t} \mathbb{E}\left[\left|\rho^{i, n}-\bar{\rho}^{i}\right|_{s}^{*}\right] d s+\left(3 K_{1}+1\right) \int_{0}^{t} \mathbb{E}\left[\left|\rho^{k, B}-\bar{\rho}^{k, B}\right|_{s}^{*}\right] d s \\
& +\int_{0}^{t} \mathbb{E}\left[\left|f^{B}\left(\bar{\rho}_{s}^{k, B}-\bar{A}_{s}^{n, m}\right)\left(\bar{\rho}_{s}^{k, B}-\bar{A}_{s}^{n, m}\right)-f^{B}\left(\bar{\rho}_{s}^{k, B}-\nu_{s}^{n}-\mathbb{E}\left[\tilde{\rho}_{s}^{i}\right]\right)\left(\bar{\rho}_{s}^{k, B}-\nu_{s}-\mathbb{E}\left[\tilde{\rho}_{s}^{i}\right]\right)\right|\right] d s \\
& \quad+\int_{0}^{t} \mathbb{E}\left[\left|\frac{1}{n-1} \sum_{j=1, j \neq i}^{n} f^{P}\left(\bar{\rho}_{s}^{j}-\bar{A}_{s}^{n, m}\right)\left(\bar{\rho}_{s}^{j}-\bar{A}_{s}^{n, m}\right)-\mathbb{E}\left[f^{P}\left(\tilde{\rho}_{s}^{i}-\mathbb{E}\left[\tilde{\rho}_{s}^{i}\right]\right)\left(\tilde{\rho}_{s}^{i}-\mathbb{E}\left[\tilde{\rho}_{s}^{i}\right]\right)\right]\right|\right] d s \\
& \quad+\int_{0}^{t} \mathbb{E}\left[\left|\bar{A}_{s}^{n, m}-\mathbb{E}\left[\tilde{\rho}_{s}^{i}\right]-\nu_{s}\right|\right] d s, \quad t \geq 0 .
\end{aligned}
$$

Proceeding as before, we find

$$
\begin{aligned}
& \mathbb{E}\left[\left|\rho^{k, B}-\bar{\rho}^{k, B}\right|_{t}^{*}\right] \\
& \leq\left(3 K_{1}+1\right) \int_{0}^{t} \mathbb{E}\left[\left|\rho^{i, n}-\bar{\rho}^{i}\right|_{s}^{*}\right] d s+\left(3 K_{1}+2\right) \int_{0}^{t} \mathbb{E}\left[\left|\rho^{k, B}-\bar{\rho}^{k, B}\right|_{s}^{*}\right] d s \\
& +\int_{0}^{t} \mathbb{E}\left[\left|f^{B}\left(\bar{\rho}_{s}^{k, B}-\bar{A}_{s}^{n, m}\right)\left(\bar{\rho}_{s}^{k, B}-\bar{A}_{s}^{n, m}\right)-f^{B}\left(\bar{\rho}_{s}^{k, B}-\nu_{s}^{n}-\mathbb{E}\left[\tilde{\rho}_{s}^{i}\right]\right)\left(\bar{\rho}_{s}^{k, B}-\nu_{s}-\mathbb{E}\left[\tilde{\rho}_{s}^{i}\right]\right)\right|\right] d s \\
& \quad+\int_{0}^{t} \mathbb{E}\left[\left|\frac{1}{n} \sum_{i=1}^{n} f^{P}\left(\bar{\rho}_{s}^{i}-\bar{A}_{s}^{n, m}\right)\left(\bar{\rho}_{s}^{i}-\bar{A}_{s}^{n, m}\right)-\mathbb{E}\left[f^{P}\left(\tilde{\rho}_{s}^{i}-\mathbb{E}\left[\tilde{\rho}_{s}^{i}\right]\right)\left(\tilde{\rho}_{s}^{i}-\mathbb{E}\left[\tilde{\rho}_{s}^{i}\right]\right)\right]\right|\right] d s
\end{aligned}
$$




$$
+\int_{0}^{t} \mathbb{E}\left[\mid \bar{A}_{s}^{n, m}-\nu_{s}-\mathbb{E}\left[\tilde{\nu}_{s}^{i} \mid\right]\right] d s,
$$

so that, summing up A.6 and A.7), we have

$$
\begin{aligned}
& \mathbb{E}\left[\left|\rho^{i, n}-\bar{\rho}^{i}\right|_{t}^{*}\right]+\mathbb{E}\left[\left|\rho^{k, B}-\bar{\rho}^{k, B}\right|_{t}^{*}\right] \\
& \leq\left(6 K_{1}+3\right) \int_{0}^{t} \mathbb{E}\left[\left|\rho^{i, n}-\bar{\rho}^{i}\right|_{s}^{*}\right] d s+\left(6 K_{1}+3\right) \int_{0}^{t} \mathbb{E}\left[\left|\rho^{k, B}-\bar{\rho}^{k, B}\right|_{s}^{*}\right] d s \\
& +2 \int_{0}^{t} \mathbb{E}\left[\left|f^{B}\left(\bar{\rho}_{s}^{k, B}-\bar{A}_{s}^{n, m}\right)\left(\bar{\rho}_{s}^{k, B}-\bar{A}_{s}^{n, m}\right)-f^{B}\left(\bar{\rho}_{s}^{k, B}-\nu_{s}^{n}-\mathbb{E}\left[\tilde{\rho}_{s}^{i}\right]\right)\left(\bar{\rho}_{s}^{k, B}-\nu_{s}-\mathbb{E}\left[\tilde{\rho}_{s}^{i}\right]\right)\right|\right] d s \\
& +\int_{0}^{t} \mathbb{E}\left[\left|\frac{1}{n-1} \sum_{j=1, j \neq i}^{n} f^{P}\left(\bar{\rho}_{s}^{j}-\bar{A}_{s}^{n, m}\right)\left(\bar{\rho}_{s}^{j}-\bar{A}_{s}^{n, m}\right)-\mathbb{E}\left[f^{P}\left(\tilde{\rho}_{s}^{i}-\mathbb{E}\left[\tilde{\rho}_{s}^{i}\right]\right)\left(\tilde{\rho}_{s}^{i}-\mathbb{E}\left[\tilde{\rho}_{s}^{i}\right]\right)\right]\right|\right] d s \\
& \quad+\int_{0}^{t} \mathbb{E}\left[\left|\frac{1}{n} \sum_{i=1}^{n} f^{P}\left(\bar{\rho}_{s}^{i}-\bar{A}_{s}^{n, m}\right)\left(\bar{\rho}_{s}^{i}-\bar{A}_{s}^{n, m}\right)-\mathbb{E}\left[f^{P}\left(\tilde{\rho}_{s}^{i}-\mathbb{E}\left[\tilde{\rho}_{s}^{i}\right]\right)\left(\tilde{\rho}_{s}^{i}-\mathbb{E}\left[\tilde{\rho}_{s}^{i}\right]\right)\right]\right|\right] d s \\
& \quad+2 \int_{0}^{t} \mathbb{E}\left[\mid \bar{A}_{s}^{n, m}-\nu_{s}-\mathbb{E}\left[\tilde{\nu}_{s}^{i} \mid\right]\right] d s, \quad t \geq 0 .
\end{aligned}
$$

We can now apply Gronwall's Lemma and obtain

$$
\begin{aligned}
& \mathbb{E}\left[\left|\rho^{i, n}-\bar{\rho}^{i}\right|_{t}^{*}\right]+\mathbb{E}\left[\left|\rho_{t}^{k, B}-\bar{\rho}_{t}^{k, B}\right|_{s}^{*}\right] \\
& \leq e^{\left(6 K_{1}+3\right) t} \int_{0}^{t} \mathbb{E}\left[\left|\frac{1}{n-1} \sum_{j=1, j \neq i}^{n} f^{P}\left(\bar{\rho}_{s}^{j}-\bar{A}_{s}^{n, m}\right)\left(\bar{\rho}_{s}^{j}-\bar{A}_{s}^{n, m}\right)-\mathbb{E}\left[f^{P}\left(\tilde{\rho}_{s}^{i}-\mathbb{E}\left[\tilde{\rho}_{s}^{i}\right]\right)\left(\tilde{\rho}_{s}^{i}-\mathbb{E}\left[\tilde{\rho}_{s}^{i}\right]\right)\right]\right|\right] d s \\
& +e^{\left(6 K_{1}+3\right) t} \int_{0}^{t} \mathbb{E}\left[\left|\frac{1}{n} \sum_{i=1}^{n} f^{P}\left(\bar{\rho}_{s}^{i}-\bar{A}_{s}^{n, m}\right)\left(\bar{\rho}_{s}^{i}-\bar{A}_{s}^{n, m}\right)-\mathbb{E}\left[f^{P}\left(\tilde{\rho}_{s}^{i}-\mathbb{E}\left[\tilde{\rho}_{s}^{i}\right]\right)\left(\tilde{\rho}_{s}^{i}-\mathbb{E}\left[\tilde{\rho}_{s}^{i}\right]\right)\right]\right|\right] d s \\
& +2 e^{\left(6 K_{1}+3\right) t} \int_{0}^{t} \mathbb{E}\left[\left|f^{B}\left(\bar{\rho}_{s}^{k, B}-\bar{A}_{s}^{n, m}\right)\left(\bar{\rho}_{s}^{k, B}-\bar{A}_{s}^{n, m}\right)-f^{B}\left(\bar{\rho}_{s}^{k, B}-\nu_{s}-\mathbb{E}\left[\tilde{\rho}_{s}^{i}\right]\right)\left(\bar{\rho}_{s}^{k, B}-\nu_{s}-\mathbb{E}\left[\tilde{\rho}_{s}^{i}\right]\right)\right|\right] d s \\
& +2 e^{\left(6 K_{1}+3\right) t} \int_{0}^{t} \mathbb{E}\left[\mid \bar{A}_{s}^{n, m}-\nu_{s}-\mathbb{E}\left[\tilde{\nu}_{s}^{i} \mid\right]\right] d s, \quad t \geq 0 .
\end{aligned}
$$

We can write

$$
\begin{aligned}
\int_{0}^{t} \mathbb{E} & {\left[\left|\frac{1}{n-1} \sum_{j=1, j \neq i}^{n} f^{P}\left(\bar{\rho}_{s}^{j}-\bar{A}_{s}^{n, m}\right)\left(\bar{\rho}_{s}^{j}-\bar{A}_{s}^{n, m}\right)-\mathbb{E}\left[f^{P}\left(\tilde{\rho}_{s}^{i}-\mathbb{E}\left[\tilde{\rho}_{s}^{i}\right]\right)\left(\tilde{\rho}_{s}^{i}-\mathbb{E}\left[\tilde{\rho}_{s}^{i}\right]\right)\right]\right|\right] d s } \\
\leq & \left(\frac{1}{n-1}-\frac{1}{n}\right) \int_{0}^{t} \mathbb{E}\left[\left|\sum_{j=1, j \neq i}^{n} f^{P}\left(\bar{\rho}_{s}^{j}-\bar{A}_{s}^{n, m}\right)\left(\bar{\rho}_{s}^{j}-\bar{A}_{s}^{n, m}\right)\right|\right] d s \\
& +\int_{0}^{t} \mathbb{E}\left[\left|\frac{1}{n} \sum_{i=1}^{n} f^{P}\left(\bar{\rho}_{s}^{i}-\bar{A}_{s}^{n, m}\right)\left(\bar{\rho}_{s}^{i}-\bar{A}_{s}^{n, m}\right)-\mathbb{E}\left[f^{P}\left(\tilde{\rho}_{s}^{i}-\mathbb{E}\left[\tilde{\rho}_{s}^{i}\right]\right)\left(\tilde{\rho}_{s}^{i}-\mathbb{E}\left[\tilde{\rho}_{s}^{i}\right]\right)\right]\right|\right] d s
\end{aligned}
$$




$$
+\frac{1}{n} \int_{0}^{t} \mathbb{E}\left[\left|f^{P}\left(\tilde{\rho}_{s}^{i}-\mathbb{E}\left[\tilde{\rho}_{s}^{i}\right]\right)\left(\tilde{\rho}_{s}^{i}-\mathbb{E}\left[\tilde{\rho}_{s}^{i}\right]\right)\right|\right] d s \quad t \geq 0
$$

with

$$
\begin{aligned}
& \left(\frac{1}{n-1}-\frac{1}{n}\right) \int_{0}^{t} \mathbb{E}\left[\left|\sum_{j=1, j \neq i}^{n} f^{P}\left(\bar{\rho}_{s}^{i}-\bar{A}_{s}^{n, m}\right)\left(\bar{\rho}_{s}^{i}-\bar{A}_{s}^{n, m}\right)\right|\right] d s \\
& \leq \frac{1}{n(n-1)} \int_{0}^{t} \sum_{j=1, j \neq i}^{n} \mathbb{E}\left[\left|f^{P}\left(\bar{\rho}_{s}^{i}-\bar{A}_{s}^{n, m}\right)\left(\bar{\rho}_{s}^{i}-\bar{A}_{s}^{n, m}\right)\right|\right] d s \\
& \quad=\frac{1}{n} \int_{0}^{t} \mathbb{E}\left[\left|f^{P}\left(\bar{\rho}_{s}^{i}-\bar{A}_{s}^{n, m}\right)\left(\bar{\rho}_{s}^{i}-\bar{A}_{s}^{n, m}\right)\right|\right] d s \leq \frac{K_{1}}{n} \int_{0}^{t} \mathbb{E}\left[\left|\bar{\rho}_{s}^{i}-\bar{A}_{s}^{n, m}\right|\right] d s, \quad t \geq 0,
\end{aligned}
$$

where the last term tends to zero when $n \rightarrow \infty$ by 3.18 .

Since it can be shown, for $t \geq 0$, that

$\lim _{n \rightarrow \infty} \int_{0}^{t} \mathbb{E}\left[\left|f^{B}\left(\bar{\rho}_{s}^{k, B}-\bar{A}_{s}^{n, m}\right)\left(\bar{\rho}_{s}^{k, B}-\bar{A}_{s}^{n, m}\right)-f^{B}\left(\bar{\rho}_{s}^{k, B}-\nu_{s}-\mathbb{E}\left[\tilde{\rho}_{s}^{i}\right]\right)\left(\bar{\rho}_{s}^{k, B}-\nu_{s}-\mathbb{E}\left[\tilde{\rho}_{s}^{i}\right]\right)\right|\right] d s=0$,

and

$$
\lim _{n \rightarrow \infty} \int_{0}^{t} \mathbb{E}\left[\mid \bar{A}_{s}^{n, m}-\nu_{s}-\mathbb{E}\left[\tilde{\nu}_{s}^{i} \mid\right]\right] d s=0, \quad t \geq 0,
$$

with the same proof as for (3.18), then by $(3.14)$ we obtain the result for $t \in[0, \delta)$.

Second step: case $t \in[\delta, 2 \delta)$.

For every $i=1, \ldots, n$ and $t \geq \delta$, we have

$$
\left|\rho_{t}^{i, n}-\bar{\rho}_{t}^{i}\right| \leq\left|\int_{0}^{\delta}\left(\rho_{s}^{i, n}-\bar{\rho}_{s}^{i}\right) d s+\int_{\delta}^{t} \Delta_{s}^{\delta, n} d s\right|
$$

where

$$
\begin{aligned}
\Delta_{s}^{\delta, n}= & \frac{1}{n-1} \sum_{j=1, j \neq i}^{n} f^{P}\left(\rho_{s-\delta}^{j, n}-A_{s-\delta}^{n, m}\right)\left(\rho_{s}^{j, n}-A_{s}^{n, m}\right)-\mathbb{E}\left[f^{P}\left(\tilde{\rho}_{t-\delta}^{i}-\mathbb{E}\left[\tilde{\rho}_{t-\delta}^{i}\right]\right)\left(\tilde{\rho}_{t}^{i}-\mathbb{E}\left[\tilde{\rho}_{t}^{i}\right]\right)\right] \\
& +\frac{1}{m} \sum_{k=1}^{m}\left(f^{B}\left(\rho_{s-\delta}^{k, B}-A_{s-\delta}^{n, m}\right)\left(\rho_{s}^{k, B}-A_{s}^{n, m}\right)-f^{B}\left(\bar{\rho}_{s-\delta}^{k, B}-\nu_{s-\delta}-\mathbb{E}\left[\tilde{\rho}_{s-\delta}^{i}\right]\right)\left(\bar{\rho}_{s}^{k, B}-\nu_{s}-\mathbb{E}\left[\tilde{\rho}_{s}^{i}\right]\right)\right) \\
& -\left(\rho_{s}^{i, n}-\bar{\rho}_{s}^{i}\right)+\left(A_{s}^{n, m}-\bar{A}_{s}^{n, m}\right)+\left(\bar{A}_{s}^{n, m}-\mathbb{E}\left[\tilde{\rho}_{s}^{i}\right]-\nu_{s} \mid\right) .
\end{aligned}
$$

Thus

$$
\left|\rho^{i, n}-\bar{\rho}^{i}\right|_{t}^{*}=\sup _{s \leq t}\left|\int_{0}^{\delta}\left(\rho_{u}^{i, n}-\bar{\rho}_{u}^{i}\right) d u+\int_{\delta}^{s} \Delta_{u}^{\delta, n} d u\right| \leq \int_{0}^{\delta}\left|\rho_{u}^{i, n}-\bar{\rho}_{u}^{i}\right| d u+\sup _{\delta \leq s \leq t} \int_{\delta}^{s}\left|\Delta_{u}^{\delta, n}\right| d u
$$




$$
=\int_{0}^{\delta}\left|\rho_{u}^{i, n}-\bar{\rho}_{u}^{i}\right| d u+\int_{\delta}^{t}\left|\Delta_{u}^{\delta, n}\right| d u, \quad \delta \leq t .
$$

For every $i=1, \ldots, n$, we have

$$
\begin{aligned}
\mathbb{E} & {\left[\int_{\delta}^{t}\left|\Delta_{s}^{\delta, n}\right| d s\right] } \\
\leq & \int_{\delta}^{t} \mathbb{E}\left[\left|\frac{1}{n-1} \sum_{j=1, j \neq i}^{n}\left(f^{P}\left(\rho_{s-\delta}^{j, n}-A_{s-\delta}^{n, m}\right)\left(\rho_{s}^{j, n}-A_{s}^{n, m}\right)-f^{P}\left(\bar{\rho}_{s-\delta}^{j}-\bar{A}_{s-\delta}^{n, m}\right)\left(\bar{\rho}_{s}^{j}-\bar{A}_{s}^{n, m}\right)\right)\right|\right] d s \\
& +\int_{\delta}^{t} \mathbb{E}\left[\left|\frac{1}{n-1} \sum_{j=1, j \neq i}^{n} f^{P}\left(\bar{\rho}_{s-\delta}^{j}-\bar{A}_{s-\delta}^{n, m}\right)\left(\bar{\rho}_{s}^{j}-\bar{A}_{s}^{n, m}\right)-\mathbb{E}\left[f^{P}\left(\tilde{\rho}_{s-\delta}^{i}-\mathbb{E}\left[\tilde{\rho}_{s-\delta}^{i}\right]\right)\left(\tilde{\rho}_{s}^{i}-\mathbb{E}\left[\tilde{\rho}_{s}^{i}\right]\right)\right]\right|\right] d s \\
& +\int_{\delta}^{t} \mathbb{E}\left[\left|\frac{1}{m} \sum_{k=1}^{m}\left(f^{B}\left(\rho_{s-\delta}^{k, B}-A_{s-\delta}^{n, m}\right)\left(\rho_{s}^{k, B}-A_{s}^{n, m}\right)-f^{B}\left(\bar{\rho}_{s-\delta}^{k, B}-\bar{A}_{s-\delta}^{n, m}\right)\left(\bar{\rho}_{s}^{k, B}-\bar{A}_{s}^{n, m}\right)\right)\right|\right] d s \\
& +\int_{\delta}^{t} \mathbb{E}\left[\frac{1}{m} \sum_{k=1}^{m}\left|f^{B}\left(\bar{\rho}_{s-\delta}^{k, B}-\bar{A}_{s-\delta}^{n, m}\right)\left(\bar{\rho}_{s}^{k, B}-\bar{A}_{s}^{n, m}\right)-f^{B}\left(\bar{\rho}_{s-\delta}^{k, B}-\nu_{s-\delta}-\mathbb{E}\left[\tilde{\rho}_{s-\delta}^{i}\right]\right)\left(\bar{\rho}_{s}^{k, B}-\nu_{s}-\mathbb{E}\left[\tilde{\rho}_{s}^{i}\right]\right)\right|\right] d s \\
& +\int_{\delta}^{t} \mathbb{E}\left[\left|\rho_{s}^{i, n}-\bar{\rho}_{s}^{i}\right|\right] d s+\int_{0}^{t} \mathbb{E}\left[\left|A_{s}^{n, m}-\bar{A}_{s}^{n, m}\right|\right] d s+\int_{0}^{t} \mathbb{E}\left[\left|\bar{A}_{s}^{n, m}-\mathbb{E}\left[\tilde{\rho}_{s}^{i}\right]-\nu_{s}\right|\right] d s, \quad \delta \leq t .
\end{aligned}
$$

By 2.10,

$$
\begin{aligned}
\int_{\delta}^{t} \mathbb{E} & {\left[\left|\frac{1}{n-1} \sum_{j=1, j \neq i}^{n}\left(f^{P}\left(\rho_{s-\delta}^{j, n}-A_{s-\delta}^{n, m}\right)\left(\rho_{s}^{j, n}-A_{s}^{n, m}\right)-f^{P}\left(\bar{\rho}_{s-\delta}^{j}-\bar{A}_{s-\delta}^{n, m}\right)\left(\bar{\rho}_{s}^{j}-\bar{A}_{s}^{n, m}\right)\right)\right|\right] d s } \\
\leq & \frac{1}{n-1} \sum_{j=1, j \neq i}^{n} \int_{\delta}^{t} \mathbb{E}\left[\left|f^{P}\left(\rho_{s-\delta}^{j, n}-A_{s-\delta}^{n, m}\right)\left(\left(\rho_{s}^{j, n}-A_{s}^{n, m}\right)-\left(\bar{\rho}_{s}^{j}-\bar{A}_{s}^{n, m}\right)\right)\right|\right] d s \\
& +\frac{1}{n-1} \sum_{j=1, j \neq i}^{n} \int_{\delta}^{t} \mathbb{E}\left[\left|\left(\bar{\rho}_{s}^{j}-\bar{A}_{s}^{n, m}\right)\left(f^{P}\left(\rho_{s-\delta}^{j, n}-A_{s-\delta}^{n, m}\right)-f^{P}\left(\bar{\rho}_{s-\delta}^{j}-\bar{A}_{s-\delta}^{n, m}\right)\right)\right|\right] d s \\
\leq & K_{1} \int_{\delta}^{t} \mathbb{E}\left[\left|\rho_{s}^{i, n}-\bar{\rho}_{s}^{i}\right|\right] d s+K_{1} \int_{\delta}^{t} \mathbb{E}\left[\left|A_{s}^{n, m}-\bar{A}_{s}^{n, m}\right|\right] d s \\
& +\int_{\delta}^{t} \mathbb{E}\left[\left|\bar{\rho}_{s}^{i}-\bar{A}_{s}^{n, m}\right|\left|f^{P}\left(\rho_{s-\delta}^{i, n}-A_{s-\delta}^{n, m}\right)-f^{P}\left(\bar{\rho}_{s-\delta}^{i}-\bar{A}_{s-\delta}^{n, m}\right)\right|\right] d s .
\end{aligned}
$$

We have that for $\delta \leq t$

$$
\begin{aligned}
& \int_{\delta}^{t} \mathbb{E}\left[\left|\bar{\rho}_{s}^{i}-\bar{A}_{s}^{n, m}\right|\left|f^{P}\left(\rho_{s-\delta}^{i, n}-A_{s-\delta}^{n, m}\right)-f^{P}\left(\bar{\rho}_{s-\delta}^{i}-\bar{A}_{s-\delta}^{n, m}\right)\right|\right] d s \\
& \quad \leq \int_{\delta}^{t}\left(\mathbb{E}\left[\left|\bar{\rho}_{s}^{i}-\bar{A}_{s}^{n, m}\right|^{2}\right] d s\right)^{1 / 2}\left(\mathbb{E}\left[\left|f^{P}\left(\rho_{s-\delta}^{i, n}-A_{s}^{n, m}\right)-f^{P}\left(\bar{\rho}_{s-\delta}^{i}-\bar{A}_{s}^{n, m}\right)\right|^{2}\right]\right)^{1 / 2} d s
\end{aligned}
$$




$$
\begin{aligned}
& \leq\left(\int_{\delta}^{t} \mathbb{E}\left[\left|\bar{\rho}_{s}^{i}-\bar{A}_{s}^{n, m}\right|^{2}\right] d s\right)^{1 / 2}\left(\int_{\delta}^{t} \mathbb{E}\left[\left|f^{P}\left(\rho_{s-\delta}^{i, n}-A_{s-\delta}^{n, m}\right)-f^{P}\left(\bar{\rho}_{s-\delta}^{i}-\bar{A}_{s-\delta}^{n, m}\right)\right|^{2}\right] d s\right)^{1 / 2} \\
& \leq\left(\int_{\delta}^{t} \mathbb{E}\left[\left|\bar{\rho}_{s}^{i}-\bar{A}_{s}^{n, m}\right|^{2}\right] d s\right)^{1 / 2}\left(\int_{\delta}^{t} \mathbb{E}\left[\left|f^{P}\left(\rho_{s-\delta}^{i, n}-A_{s-\delta}^{n, m}\right)^{2}-f^{P}\left(\bar{\rho}_{s-\delta}^{i}-\bar{A}_{s-\delta}^{n, m}\right)^{2}\right|\right] d s\right)^{1 / 2} \\
& \leq \sqrt{2 K_{1}}\left(\int_{\delta}^{t} \mathbb{E}\left[\left|\bar{\rho}_{s}^{i}-\bar{A}_{s}^{n, m}\right|^{2}\right] d s\right)^{1 / 2}\left(\int_{\delta}^{t} \mathbb{E}\left[\left|f^{P}\left(\rho_{s-\delta}^{i, n}-A_{s-\delta}^{n, m}\right)-f^{P}\left(\bar{\rho}_{s-\delta}^{i}-\bar{A}_{s-\delta}^{n, m}\right)\right|\right] d s\right)^{1 / 2},
\end{aligned}
$$

where we have used that $|a-b|^{2} \leq\left|a^{2}-b^{2}\right|$ for $a, b \in \mathbb{R}^{+}$.

Then, setting $G_{1}^{n}(t):=\left(\int_{\delta}^{t} \mathbb{E}\left[\left|\bar{\rho}_{s}^{i}-\bar{A}_{s}^{n, m}\right|^{2}\right] d s\right)^{1 / 2}$, by A.13 we have

$$
\begin{aligned}
& \int_{\delta}^{t} \mathbb{E}\left[\left|\frac{1}{n-1} \sum_{j=1, j \neq i}^{n}\left(f^{P}\left(\rho_{s-\delta}^{j, n}-A_{s-\delta}^{n, m}\right)\left(\rho_{s}^{j, n}-A_{s}^{n, m}\right)-f^{P}\left(\bar{\rho}_{s-\delta}^{j}-\bar{A}_{s-\delta}^{n, m}\right)\left(\bar{\rho}_{s}^{j}-\bar{A}_{s}^{n, m}\right)\right)\right|\right] d s \\
& \leq K_{1} \int_{\delta}^{t} \mathbb{E}\left[\left|\rho_{s}^{i, n}-\bar{\rho}_{s}^{i}\right|\right] d s+K_{1} \int_{\delta}^{t} \mathbb{E}\left[\left|A_{s}^{n, m}-\bar{A}_{s}^{n, m}\right|\right] d s \\
& \quad+\sqrt{2 K_{1}} G_{1}^{n}(t)\left(\int_{\delta}^{t} \mathbb{E}\left[\left|f^{P}\left(\rho_{s-\delta}^{i, n}-A_{s-\delta}^{n, m}\right)-f^{P}\left(\bar{\rho}_{s-\delta}^{i}-\bar{A}_{s-\delta}^{n, m}\right)\right|\right] d s\right)^{1 / 2}, \quad \delta \leq t
\end{aligned}
$$

For $\delta \leq t<2 \delta$,

$$
\begin{aligned}
& \int_{\delta}^{t} \mathbb{E}\left[\left|f^{P}\left(\rho_{s-\delta}^{i, n}-A_{s-\delta}^{n, m}\right)-f^{P}\left(\bar{\rho}_{s-\delta}^{i}-\bar{A}_{s-\delta}^{n, m}\right)\right|\right] d s=\mathbb{E}\left[\int_{\delta}^{t}\left(\left|f^{P}\left(\rho_{s-\delta}^{i, n}-A_{s-\delta}^{n, m}\right)-f^{P}\left(\bar{\rho}_{s-\delta}^{i}-\bar{A}_{s-\delta}^{n, m}\right)\right|\right) d s\right] \\
& \quad=\mathbb{E}\left[\int_{0}^{t-\delta}\left(\left|f^{P}\left(\rho_{u}^{i, n}-A_{u}^{n, m}\right)-f^{P}\left(\bar{\rho}_{u}^{i}-\bar{A}_{u}^{n, m}\right)\right|\right) d u\right] \leq \int_{0}^{\delta} \mathbb{E}\left[\left|f^{P}\left(\rho_{u}^{i, n}-A_{u}^{n, m}\right)-f^{P}\left(\bar{\rho}_{u}^{i}-\bar{A}_{u}^{n, m}\right)\right|\right] d u
\end{aligned}
$$

thus we can rewrite A.14 as

$$
\begin{aligned}
& \int_{\delta}^{t} \mathbb{E}\left[\left|\frac{1}{n-1} \sum_{j=1, j \neq i}^{n}\left(f^{P}\left(\rho_{s-\delta}^{j, n}-A_{s-\delta}^{n, m}\right)\left(\rho_{s}^{j, n}-A_{s}^{n, m}\right)-f^{P}\left(\bar{\rho}_{s-\delta}^{j}-\bar{A}_{s-\delta}^{n, m}\right)\left(\bar{\rho}_{s}^{j}-\bar{A}_{s}^{n, m}\right)\right)\right|\right] d s \\
& \leq K_{1} \int_{\delta}^{t} \mathbb{E}\left[\left|\rho_{s}^{i, n}-\bar{\rho}_{s}^{i}\right|\right] d s+K_{1} \int_{\delta}^{t} \mathbb{E}\left[\left|A_{s}^{n, m}-\bar{A}_{s}^{n, m}\right|\right] d s \\
& \quad+\sqrt{2 K_{1}} G_{1}^{n}(t)\left(\int_{0}^{\delta} \mathbb{E}\left[\left|f^{P}\left(\rho_{s}^{i, n}-A_{s}^{n, m}\right)-f^{P}\left(\bar{\rho}_{s}^{i}-\bar{A}_{s}^{n, m}\right)\right|\right] d s\right)^{1 / 2}, \quad \delta \leq t \leq 2 \delta .
\end{aligned}
$$

Similarly,

$$
\begin{aligned}
& \int_{0}^{t} \mathbb{E}\left[\left|\frac{1}{m} \sum_{k=1}^{m}\left(f^{B}\left(\rho_{s-\delta}^{k, B}-A_{s-\delta}^{n, m}\right)\left(\rho_{s}^{k, B}-A_{s}^{n, m}\right)-f^{B}\left(\bar{\rho}_{s-\delta}^{k, B}-\bar{A}_{s-\delta}^{n, m}\right)\left(\bar{\rho}_{s}^{k, B}-\bar{A}_{s}^{n, m}\right)\right)\right|\right] d s \\
& \quad \leq K_{1} \int_{\delta}^{t} \mathbb{E}\left[\left|\rho_{s}^{k, B}-\bar{\rho}_{s}^{k, B}\right|\right] d s+K_{1} \int_{\delta}^{t} \mathbb{E}\left[\left|A_{s}^{n, m}-\bar{A}_{s}^{n, m}\right|\right] d s
\end{aligned}
$$




$$
+\sqrt{2 K_{1}} G_{2}^{n}(t)\left(\int_{0}^{\delta} \mathbb{E}\left[\left|f^{B}\left(\rho_{s}^{i, n}-A_{s}^{n, m}\right)-f^{B}\left(\bar{\rho}_{s}^{i}-\bar{A}_{s}^{n, m}\right)\right|\right] d s\right)^{1 / 2}, \quad \delta \leq t .
$$

with $G_{2}^{n}(t):=\left(\int_{\delta}^{t} \mathbb{E}\left[\left|\bar{\rho}_{s}^{k, B}-\bar{A}_{s}^{n, m}\right|^{2}\right] d s\right)^{1 / 2}$. From A.3, A.11, A.12, A.15 and A.16 we obtain

$$
\begin{aligned}
\mathbb{E} & {\left[\left|\rho^{i, n}-\bar{\rho}^{i}\right|_{t}^{*}\right] } \\
\leq & \left(3 K_{1}+2\right) \int_{\delta}^{t} \mathbb{E}\left[\left|\rho^{i, n}-\bar{\rho}^{i}\right|_{s}^{*}\right] d s+\left(3 K_{1}+1\right) \int_{\delta}^{t} \mathbb{E}\left[\left|\rho^{k, B}-\bar{\rho}^{k, B}\right|_{s}^{*}\right] d s \\
& +\sqrt{2 K_{1}} G_{1}^{n}(t)\left(\int_{0}^{\delta} \mathbb{E}\left[\left|f^{P}\left(\rho_{s}^{i, n}-A_{s}^{n, m}\right)-f^{P}\left(\bar{\rho}_{s}^{i}-\bar{A}_{s}^{n, m}\right)\right|\right] d s\right)^{1 / 2} \\
& +\sqrt{2 K_{1}} G_{2}^{n}(t)\left(\int_{0}^{\delta} \mathbb{E}\left[\left|f^{B}\left(\rho_{s}^{i, n}-A_{s}^{n, m}\right)-f^{B}\left(\bar{\rho}_{s}^{i}-\bar{A}_{s}^{n, m}\right)\right|\right] d s\right)^{1 / 2} \\
& +\int_{0}^{t} \mathbb{E}\left[\left|f^{B}\left(\bar{\rho}_{s-\delta}^{k, B}-\bar{A}_{s-\delta}^{n, m}\right)\left(\bar{\rho}_{s}^{k, B}-\bar{A}_{s}^{n, m}\right)-f^{B}\left(\bar{\rho}_{s-\delta}^{k, B}-\nu_{s-\delta}-\mathbb{E}\left[\tilde{\rho}_{s-\delta}^{i}\right]\right)\left(\bar{\rho}_{s}^{k, B}-\nu_{s}-\mathbb{E}\left[\tilde{\rho}_{s}^{i}\right]\right)\right|\right] d s \\
& +\int_{0}^{t} \mathbb{E}\left[\left|\frac{1}{n-1} \sum_{j=1, j \neq i}^{n} f^{P}\left(\bar{\rho}_{s-\delta}^{j}-\bar{A}_{s-\delta}^{n, m}\right)\left(\bar{\rho}_{s}^{j}-\bar{A}_{s}^{n, m}\right)-\mathbb{E}\left[f^{P}\left(\tilde{\rho}_{s-\delta}^{i}-\mathbb{E}\left[\tilde{\rho}_{s-\delta}^{i}\right]\right)\left(\tilde{\rho}_{s}^{i}-\mathbb{E}\left[\tilde{\rho}_{s}^{i}\right]\right)\right]\right|\right] d s \\
& +\int_{0}^{\delta} \mathbb{E}\left[\left|\rho_{s}^{i, n}-\bar{\rho}_{s}^{i}\right|\right] d s+\int_{0}^{t} \mathbb{E}\left[\left|\bar{A}_{s}^{n, m}-\mathbb{E}\left[\tilde{\rho}_{s}^{i}\right]-\nu_{s}\right|\right] d s, \quad \delta \leq t<2 \delta .
\end{aligned}
$$

At the same way, by (2.2) and (3.3) we have

$$
\begin{aligned}
\mathbb{E} & {\left[\left|\rho^{k, B}-\bar{\rho}^{k, B}\right|_{t}^{*}\right] } \\
\leq & \left(3 K_{1}+1\right) \int_{\delta}^{t} \mathbb{E}\left[\left|\rho^{i, n}-\bar{\rho}^{i}\right|_{s}^{*}\right] d s+\left(3 K_{1}+2\right) \int_{\delta}^{t} \mathbb{E}\left[\left|\rho^{k, B}-\bar{\rho}^{k, B}\right|_{s}^{*}\right] d s \\
& +\sqrt{2 K_{1}} G_{1}^{n}(t)\left(\int_{0}^{\delta} \mathbb{E}\left[\left|f^{P}\left(\rho_{s}^{i, n}-A_{s}^{n, m}\right)-f^{P}\left(\bar{\rho}_{s}^{i}-\bar{A}_{s}^{n, m}\right)\right|\right] d s\right)^{1 / 2} \\
& +\sqrt{2 K_{1}} G_{2}^{n}(t)\left(\int_{0}^{\delta} \mathbb{E}\left[\left|f^{B}\left(\rho_{s}^{i, n}-A_{s}^{n, m}\right)-f^{B}\left(\bar{\rho}_{s}^{i}-\bar{A}_{s}^{n, m}\right)\right|\right] d s\right)^{1 / 2} \\
& +\int_{0}^{t} \mathbb{E}\left[\left|f^{B}\left(\bar{\rho}_{s-\delta}^{k, B}-\bar{A}_{s-\delta}^{n, m}\right)\left(\bar{\rho}_{s}^{k, B}-\bar{A}_{s}^{n, m}\right)-f^{B}\left(\bar{\rho}_{s-\delta}^{k, B}-\nu_{s-\delta}-\mathbb{E}\left[\tilde{\rho}_{s-\delta}^{i}\right]\right)\left(\bar{\rho}_{s}^{k, B}-\nu_{s}-\mathbb{E}\left[\tilde{\rho}_{s}^{i}\right]\right)\right|\right] d s \\
& +\int_{0}^{t} \mathbb{E}\left[\left|\frac{1}{n} \sum_{i=1}^{n} f^{P}\left(\bar{\rho}_{s-\delta}^{i}-\bar{A}_{s-\delta}^{n, m}\right)\left(\bar{\rho}_{s}^{i}-\bar{A}_{s}^{n, m}\right)-\mathbb{E}\left[f^{P}\left(\tilde{\rho}_{s-\delta}^{i}-\mathbb{E}\left[\tilde{\rho}_{s-\delta}^{i}\right]\right)\left(\tilde{\rho}_{s}^{i}-\mathbb{E}\left[\tilde{\rho}_{s}^{i}\right]\right)\right]\right|\right] d s \\
& +\int_{0}^{\delta} \mathbb{E}\left[\left|\rho_{s}^{k, B}-\bar{\rho}_{s}^{k, B}\right|\right] d s+\int_{0}^{t} \mathbb{E}\left[\left|\bar{A}_{s}^{n, m}-\nu_{s}-\mathbb{E}\left[\tilde{\nu}_{s}^{i}\right]\right|\right] d s, \quad \delta \leq t<2 \delta .
\end{aligned}
$$

Summing up (A.17) and (A.18) we find $\mathbb{E}\left[\left|\rho^{i, n}-\bar{\rho}^{i}\right|_{t}^{*}\right]+\mathbb{E}\left[\left|\rho^{k, B}-\bar{\rho}^{k, B}\right|_{t}^{*}\right]$ 


$$
\begin{aligned}
& \leq\left(6 K_{1}+3\right) \int_{0}^{t}\left(\mathbb{E}\left[\left|\rho^{i, n}-\bar{\rho}^{i}\right|_{s}^{*}\right]+\mathbb{E}\left[\left|\rho^{k, B}-\bar{\rho}^{k, B}\right|_{s}^{*}\right]\right) d s \\
& +2 \sqrt{2 K_{1}} G_{1}^{n}(t)\left(\int_{0}^{\delta} \mathbb{E}\left[\left|f^{P}\left(\rho_{s}^{i, n}-A_{s}^{n, m}\right)-f^{P}\left(\bar{\rho}_{s}^{i}-\bar{A}_{s}^{n, m}\right)\right|\right] d s\right)^{1 / 2} \\
& +2 \sqrt{2 K_{1}} G_{2}^{n}(t)\left(\int_{0}^{\delta} \mathbb{E}\left[\left|f^{B}\left(\rho_{s}^{i, n}-A_{s}^{n, m}\right)-f^{B}\left(\bar{\rho}_{s}^{i}-\bar{A}_{s}^{n, m}\right)\right|\right] d s\right)^{1 / 2} \\
& +\int_{0}^{t} \mathbb{E}\left[\left|f^{B}\left(\bar{\rho}_{s-\delta}^{k, B}-\bar{A}_{s-\delta}^{n, m}\right)\left(\bar{\rho}_{s}^{k, B}-\bar{A}_{s}^{n, m}\right)-f^{B}\left(\bar{\rho}_{s-\delta}^{k, B}-\nu_{s-\delta}-\mathbb{E}\left[\tilde{\rho}_{s-\delta}^{i}\right]\right)\left(\bar{\rho}_{s}^{k, B}-\nu_{s}-\mathbb{E}\left[\tilde{\rho}_{s}^{i}\right]\right)\right|\right] d s \\
& +\int_{0}^{t} \mathbb{E}\left[\left|\frac{1}{n-1} \sum_{j=1, j \neq i}^{n} f^{P}\left(\bar{\rho}_{s-\delta}^{j}-\bar{A}_{s-\delta}^{n, m}\right)\left(\bar{\rho}_{s}^{j}-\bar{A}_{s}^{n, m}\right)-\mathbb{E}\left[f^{P}\left(\tilde{\rho}_{s-\delta}^{i}-\mathbb{E}\left[\tilde{\rho}_{s-\delta}^{i}\right]\right)\left(\tilde{\rho}_{s}^{i}-\mathbb{E}\left[\tilde{\rho}_{s}^{i}\right]\right)\right]\right|\right] d s \\
& +\int_{0}^{t} \mathbb{E}\left[\left|\frac{1}{n} \sum_{i=1}^{n} f^{P}\left(\bar{\rho}_{s-\delta}^{i}-\bar{A}_{s-\delta}^{n, m}\right)\left(\bar{\rho}_{s}^{i}-\bar{A}_{s}^{n, m}\right)-\mathbb{E}\left[f^{P}\left(\tilde{\rho}_{s-\delta}^{i}-\mathbb{E}\left[\tilde{\rho}_{s-\delta}^{i}\right]\right)\left(\tilde{\rho}_{s}^{i}-\mathbb{E}\left[\tilde{\rho}_{s}^{i}\right]\right)\right]\right|\right] d s \\
& +2 \int_{0}^{t} \mathbb{E}\left[\mid \bar{A}_{s}^{n, m}-\nu_{s}-\mathbb{E}\left[\tilde{\nu}_{s}^{i} \mid\right]\right] d s, \quad \delta \leq t<2 \delta .
\end{aligned}
$$

With the same computations used in the first step of the proof, we show that the last four terms of A.21 converge to zero when $n \rightarrow \infty$ by the proof of Proposition 3.3 . It remains to show that A.19) and A.20 tend to zero. We write

$$
\begin{aligned}
& \int_{0}^{\delta} \mathbb{E}\left[\left|f^{P}\left(\rho_{s}^{i, n}-A_{s}^{n, m}\right)-f^{P}\left(\bar{\rho}_{s}^{i}-\bar{A}_{s}^{n, m}\right)\right|\right] d s \\
& \leq \int_{0}^{\delta} \mathbb{E}\left[\left|f^{P}\left(\rho_{s}^{i, n}-A_{s}^{n, m}\right)-f^{P}\left(\bar{\rho}_{s}^{i}-\nu_{s}-\mathbb{E}\left[\tilde{\rho}_{s}^{i}\right]\right)\right|\right] d s \\
& +\int_{0}^{\delta} \mathbb{E}\left[\left|f^{P}\left(\bar{\rho}_{s}^{i}-\nu_{s}-\mathbb{E}\left[\tilde{\rho}_{s}^{i}\right]\right)-f^{P}\left(\bar{\rho}_{s}^{i}-\bar{A}_{s}^{n, m}\right)\right|\right] d s .
\end{aligned}
$$

We now show that the terms in $\mathrm{A} .22$ tend to 0 by the dominated convergence theorem. To this purpose, we first note that we have

$$
\begin{aligned}
\int_{0}^{\delta} & \mathbb{E}\left[\left|\rho_{s}^{i, n}-A_{s}^{n, m}-\left(\bar{\rho}_{s}^{i}-\nu_{s}-\mathbb{E}\left[\tilde{\rho}_{s}^{i}\right]\right)\right|\right] d s \leq \int_{0}^{\delta} \mathbb{E}\left[\left|\rho_{s}^{i, n}-\bar{\rho}_{s}^{i}\right|\right] d s+\int_{0}^{\delta} \mathbb{E}\left[\left|A_{s}^{n, m}-\nu_{s}-\mathbb{E}\left[\tilde{\rho}_{s}^{i}\right]\right|\right] d s \\
\leq & \int_{0}^{\delta} \mathbb{E}\left[\left|\rho_{s}^{i, n}-\bar{\rho}_{s}^{i}\right|\right] d s+\int_{0}^{\delta} \mathbb{E}\left[\left|A_{s}^{n, m}-\bar{A}_{s}^{n, m}\right|\right] d s+\int_{0}^{\delta} \mathbb{E}\left[\left|\bar{A}_{s}^{n, m}-\nu_{s}-\mathbb{E}\left[\tilde{\rho}_{s}^{i}\right]\right|\right] d s \\
\leq & 2 \int_{0}^{\delta} \mathbb{E}\left[\left|\rho_{s}^{i, n}-\bar{\rho}_{s}^{i}\right|\right] d s+\int_{0}^{\delta} \mathbb{E}\left[\left|\rho_{s}^{k, B}-\bar{\rho}_{s}^{k, B}\right|\right] d s+\int_{0}^{\delta} \mathbb{E}\left[\left|\bar{A}_{s}^{n, m}-\nu_{s}-\mathbb{E}\left[\tilde{\rho}_{s}^{i}\right]\right|\right] d s
\end{aligned}
$$

by A.3. By the first step of the proof, the first two integrals above tend to zero when $n \rightarrow \infty$, since

$$
\int_{0}^{\delta} \mathbb{E}\left[\left|\rho_{s}^{i, n}-\bar{\rho}_{s}^{i}\right|\right] d s \leq \int_{0}^{\delta} \mathbb{E}\left[\left|\rho^{i, n}-\bar{\rho}^{i}\right|_{\delta}^{*}\right] d s=\delta \mathbb{E}\left[\left|\rho^{i, n}-\bar{\rho}^{i}\right|_{\delta}^{*}\right]
$$


whereas

$$
\lim _{n \rightarrow \infty} \int_{0}^{\delta} \mathbb{E}\left[\left|\bar{A}_{s}^{n, m}-\nu_{s}-\mathbb{E}\left[\tilde{\rho}_{s}^{i}\right]\right|\right] d s=0
$$

by A.10. Moreover,

$$
\int_{0}^{\delta} \mathbb{E}\left[\left|\bar{\rho}_{s}^{i}-\nu_{s}-\mathbb{E}\left[\tilde{\rho}_{s}^{i}\right]-\left(\bar{\rho}_{s}^{i}-\bar{A}_{s}^{n, m}\right)\right|\right] d s=\int_{0}^{\delta} \mathbb{E}\left[\left|\bar{A}_{s}^{n, m}-\nu_{s}-\mathbb{E}\left[\tilde{\rho}_{s}^{i}\right]\right| d s,\right.
$$

which goes to zero when $n \rightarrow \infty$ as shown above.

We have then proved that for all $m,\left(\rho_{s}^{i, n}-A_{s}^{n, m}\right)_{n \in \mathbb{N}}$ and $\left(\bar{\rho}_{s}^{i, n}-\bar{A}_{s}^{n, m}\right)_{n \in \mathbb{N}}$ convergence to $\bar{\rho}_{s}^{i}-\nu_{s}-\mathbb{E}\left[\tilde{\rho}_{s}^{i}\right]$ in $L^{1}([0, \delta] \times \Omega, d t \otimes P)$. This implies that for all $m,\left(\rho_{s}^{i, n}-A_{s}^{n, m}\right)_{n \in \mathbb{N}}$ and $\left(\bar{\rho}_{s}^{i, n}-\bar{A}_{s}^{n, m}\right)_{n \in \mathbb{N}}$ converge to $\bar{\rho}_{s}^{i}-\nu_{s}-\mathbb{E}\left[\tilde{\rho}_{s}^{i}\right]$ in measure with respect to $d t \otimes P$ on $[0, \delta] \times \Omega$.

By the continuous mapping theorem, since $f^{P}$ is continuous it follows that $\left(f^{P}\left(\rho_{s}^{i, n}-A_{s}^{n, m}\right)\right)_{n \in \mathbb{N}}$ and $\left(f^{P}\left(\bar{\rho}_{s}^{i, n}-\bar{A}_{s}^{n, m}\right)\right)_{n \in \mathbb{N}}$ converge to $f^{P}\left(\bar{\rho}_{s}^{i}-\nu_{s}-\mathbb{E}\left[\tilde{\rho}_{s}^{i}\right]\right)$ in measure with respect to $d t \otimes P$ on $[0, \delta] \times \Omega$. By $(2.10)$, we can apply the dominated convergence theorem, see Theorem 2 in Chapter 6 of Chow and Teicher [23], and obtain that

$$
\int_{0}^{\delta} \mathbb{E}\left[\left|f^{P}\left(\rho_{s}^{i, n}-A_{s}^{n, m}\right)-f^{P}\left(\bar{\rho}_{s}^{i}-\nu_{s}-\mathbb{E}\left[\tilde{\rho}_{s}^{i}\right]\right)\right|\right] d s \underset{n \rightarrow \infty}{\longrightarrow} 0
$$

and

$$
\int_{0}^{\delta} \mathbb{E}\left[\left|f^{P}\left(\bar{\rho}_{s}^{i}-\nu_{s}-\mathbb{E}\left[\tilde{\rho}_{s}^{i}\right]\right)-f^{P}\left(\bar{\rho}_{s}^{i}-\bar{A}_{s}^{n, m}\right)\right|\right] d s \underset{n \rightarrow \infty}{\longrightarrow} 0 .
$$

Hence A.19 converges to zero when $n \rightarrow \infty$, by A.22. Analogously, we can prove the same for $(\mathrm{A} .20)$.

Then applying Gronwall's Lemma to A.21 we prove the result for $t \in[\delta, 2 \delta)$. The result then follows by proceeding in the same way for all the steps $t \in[k \delta,(k+1) \delta), k \geq 2$.

\section{Acknowledgments}

The authors would like to thank three anonymous referees and the associated editor for their careful reading and useful comments, which have contributed to improve the quality of the paper.

\section{References}

[1] D. Abreu and M.K. Brunnermeier. Bubbles and crashes. Econometrica, 71(1):173-204, 2003.

[2] F. Allen and D. Gale. Bubbles and crisis. The Economic Journal, 110:236-255, 2000. 
[3] H. Amini and A. Minca. Inhomogeneous financial networks and contagious links. Operations Research, 64(5):1109-1120, 2016.

[4] H. Amini, R. Cont, and A. Minca. Stress testing the resilience of financial networks. International Journal of Theoretical and Applied Finance, 15(1), 2012.

[5] H. Amini, R. Cont, and A. Minca. Resilience to contagion in financial networks. Mathematical Finance, 26(2):329-365, 2016.

[6] P. Battiston. Rational bubbles in closed economies. Preprint, 2015.

[7] S. Battiston, D. Delli Gatti, M. Gallegati, B.C. Greenwald, and J.E. Stiglitz. Liaisons dangerouses: increasing connectivity, risk sharing, and systemic risk. Journal of Economic Dynamics and Control, 36(8):1121-1141, 2012.

[8] F. Biagini, J.P. Fouque, M. Frittelli, and T. Meyer-Brandis. A unified approach to systemic risk measures via acceptance sets. Mathematical Finance, 2017.

[9] F. Biagini, J.P. Fouque, M. Frittelli, and T. Meyer-Brandis. On fairness of systemic risk measures. Preprint, LMU, 2018.

[10] F. Biagini, A. Mazzon, and T. Meyer-Brandis. Liquidity induced asset bubbles via flows of ELMMs. SIAM Journal on Financial Mathematics, 9(2):800-834, 2018.

[11] G. Bianconi and A.L. Barabàsi. Competition and multiscaling in evolving networks. Europhys, Lett, 54(4):436-442, 2001.

[12] D. Bisias, M. Flood, A. W. Lo, and S. Valavanis. A survey of systemic risk analytics. Annual Review of Economics, 4(1):255-296, 2012.

[13] L. Bo and A. Capponi. Systemic risk in interbanking networks. SIAM Journal on Financial Mathematics, 6(1):386-424, 2015.

[14] M.K. Brunnermeier. Bubbles. New Palgrave Dictionary of Economics, Second Edition, 2008.

[15] M.K. Brunnermeier and M. Oehmke. Bubbles, financial crises, and systemic risk. In Handbook of the Economics of Finance, volume 2, pages 1221-1288. Elsevier, 2013.

[16] M.K. Brunnermeier and I. Schnabel. Bubbles and central banks: Historical perspectives. 2015.

[17] R. Carmona, J.-P. Fouque, and L.-H. Sun. Mean field games and systemic risk. Communications in Mathematical Sciences, 13(4):911-933, 2015. 
[18] R. Carmona, J.-P. Fouque, S.M. Mousavi, and L.-H. Sun. Systemic risk and stochastic games with delay. Journal of Optimization Theory and Applications, pages 1-34, 2016.

[19] C. Chen, G. Iyengar, and C. C. Moallemi. An axiomatic approach to systemic risk. Management Science, 59(6):1373-1388, 2013.

[20] Y. Choi and R. Douady. Chaos and Bifurcation in 2007-08 Financial Crisis. Management Science, 2011.

[21] Youngna Choi and Raphael Douady. Financial crisis and contagion: A dynamical systems approach. Handbook on systemic risk, page 453, 2013.

[22] C. Chong and C. Klüppelberg. Partial mean field limits in heterogeneous networks. Preprint, 2015.

[23] Y. S. Chow and H. Teicher. Probability theory: independence, interchangeability, martingales. Springer Science \& Business Media, 2012.

[24] A.M.G. Cox and D.G. Hobson. Local martingales, bubbles and option prices. Finance Stochastics, 9(4):477-492, 2005.

[25] J.B. DeLong, A. Shleifer, L. Summers, and R. Waldmann. Noise trader risk in financial markets. Journal of Political Economy, 98(4):703?738, 1990.

[26] N. Detering, T. Meyer-Brandis, and K. Panagiotou. Bootstrap percolation in directed and inhomogeneous random graphs. Preprint, LMU, 2015.

[27] N. Detering, T. Meyer-Brandis, K. Panagiotou, and D. Ritter. Managing default contagion in inhomogeneous financial networks. Preprint, LMU, 2017.

[28] N. Detering, T. Meyer-Brandis, K. Panagiotou, and D. Ritter. Financial contagion in a generalized stochastic block model. Preprint, LMU, 2018.

[29] S. Drapeau, M. Kupper, E.R. Gianin, L. Tangpi, et al. Dual representation of minimal supersolutions of convex BSDEs. In Annales de l'Institut Henri Poincaré, Probabilités et Statistiques, volume 52, pages 868-887. Institut Henri Poincaré, 2016.

[30] P.E. Earl, T-C Peng, and J. Potts. Decision-rule cascades and the dynamics of speculative bubbles. Journal of Economic Psychology, 28:351-364, 2007.

[31] L. Eisenberg and T.H. Noe. Systemic risk in financial systems. Management Science, 47(2):236-249, 2001. 
[32] M. Elliott, B Golub, and M.O. Jackson. Financial networks and contagion. American Economic Review, 104(10):3115-3153, 2014.

[33] F. Fang, Y. Sun, and K. Spiliopoulos. On the effect of heterogeneity on flocking behavior and systemic risk. Statistics \& Risk Modeling, 34(3-4):141-155, 2017.

[34] Z. Feinstein, B. Rudloff, and S. Weber. Measures of systemic risk. SIAM Journal on Financial Mathematics, 8(1):672-708, 2017.

[35] H. Föllmer. Equilibria in financial markets with heterogeneous agents: A probabilistic perspective. Journal of Mathematical Economics, 41(1-2):123-155, 2005.

[36] J.-P. Fouque and T. Ichiba. Stability in a model of interbank lending. SIAM Journal on Financial Mathematics, 4(1):784-803, 2013.

[37] J.-P. Fouque and L.-H. Sun. Systemic risk illustrated. In A. Palczewski and L. Stettner: Advances in Mathematics of Finance. Cambridge, 2013.

[38] P. Gai and S. Kapadia. Contagion in financial networks. Proceedings of the Royal Society, 466:2401-2423, 2010.

[39] J. Garnier, G. Papanicolau, and T-W. Yang. Diversification in financial networks may increase systemic risk, pages 423-443. Cambridge University Press, 2013.

[40] J. Garnier, G. Papanicolau, and T-W. Yang. Large deviations for a mean field model of systemic risk. SIAM Journal on Financial Mathematics, 4(1):151-184, 2013.

[41] B. Hambly, S. Ledger, and A. Sojmark. A McKean-Vlasov equation with positive feedback and blow-ups. arXiv:1801.07703, 2018.

[42] J.M. Harrison and D.M. Kreps. Speculative investor behavior in a stock market with heterogeneous expectations. The Quarterly Journal of Economics, 92(2):323-336, 1978.

[43] H. Hoffmann, T. Meyer-Brandis, and G. Svindland. Risk-consistent conditional systemic risk measures. Stochastic Processes and their Applications, 126(7):2014-2037, 2016.

[44] H. Hoffmann, T. Meyer-Brandis, and G. Svindland. Allocation of systemic risk. Preprint, LMU, 2016.

[45] J. Hull and A White. Valuing credit default swaps II: Modeling default correlations. Journal of Derivatives, 8(3):12-22, 2001.

[46] T.R. Hurd. Contagion! Systemic Risk in Financial Networks. Springer, 2016. 
[47] R. Jarrow and P. Protter. Forward and futures prices with bubbles. International Journal of Theoretical and Applied Finance, 12(7):901-924, 2009.

[48] R. Jarrow and P. Protter. Foreign currency bubbles. Review of Derivatives Research, 14(1):67-83, 2011.

[49] R. Jarrow, P. Protter, and K. Shimbo. Asset price bubbles in complete markets. Advances in Mathematical Finance, In Honor of Dilip B. Madan:105-130, 2007.

[50] R. Jarrow, P. Protter, and K. Shimbo. Asset price bubbles in incomplete markets. Mathematical Finance, 20(2):145-185, 2010.

[51] R. Jarrow, Y. Kchia, and P. Protter. How to detect an asset bubble. SIAM Journal on Financial Mathematics, 2:839-865, 2011.

[52] R. Jarrow, P. Protter, and A. Roch. A Liquidity Based Model for Asset Price. Quantitative Finance, 12(1):1339-1349, 2012.

[53] T. Kaizoji. Speculative bubbles and crashes in stock markets: an interacting-agent model of speculative activity. Phisica A, 287:493-506, 2000.

[54] I. Karatzas and S.E. Shreve. Brownian Motion and Stochastic Calculus, volume 113 of Graduate Texts in Mathematics. Springer-Verlag, New York, second edition, 1991.

[55] O. Kley, O.C. Klüppelberg, and L. Reichel. Systemic risk through contagion in a coreperiphery structured banking network. In Polen Banach Center Publications, Warschau, editor, A. Palczewski and L. Stettner: Advances in Mathematics of Finance, volume 104, 2015.

[56] E. Kromer, L. Overbeck, and K. Zilch. Systemic risk measures on general measurable spaces. Mathematical Methods of Operations Research, 84(2):323-357, 2016.

[57] M. Loewenstein and G.A. Willard. Rational equilibrium asset-pricing bubbles in continuous trading models. Journal of Economic Theory, 91(1):17-58, 2000.

[58] A. Maheshwari and A. Sarantsev. A model of interbank flows, borrowing, and investing. Risks, 6(4):134, 2017.

[59] X. Mao. Stochastic differential equations and applications, second edition. Horwood Publishing Chichester, UK, 2007.

[60] A. Mazzon. Financial asset bubbles in Networks. PhD thesis, 2018. URL https://www. fm.mathematik. uni-muenchen.de/download/publications/phdthesismazzon.pdf. 
[61] H.P. McKean. A class of Markov processes associated with nonlinear parabolic equations. Proceedings of the National Academy of Sciences, 56(6):1907-1911, 1966.

[62] H.P. McKean. Speed of approach to equilibrium for Kac's caricature of a Maxwellian gas. Archive for Rational Mechanics and Analysis, 21(5):343-367, 1966.

[63] E.M. Miller. Risk, uncertainty, and divergence of opinion. The Journal of Finance, 32 (4):1151-1168, 1977.

[64] R. Pastor-Satorras and A. Vespignani. Epidemic dynamics and endemic states in complex networks. Phys. Rev. E., 63(066117), 2001.

[65] R. Pastor-Satorras and A. Vespignani. Epidemic spreading in scale-free networks. Phys. Rev. Lett., 86:3200-3203, 2001.

[66] P. Protter. Stochastic integration and differential equations, Second Edition. SpringerVerlag, Berlin, 2005.

[67] P. Protter. A mathematical theory of financial bubbles, volume 2081 of Lecture Notes in Mathematics of $V$. Henderson and R. Sincar editors, Paris-Princeton Lectures on Mathematical Finance. Springer, 2013.

[68] J. Scheinkman and W. Xiong. Overconfidence and speculative bubbles. Journal of political economy, 111(6):1183-1219, 2003.

[69] J. Scheinkman and W. Xiong. Speculation, trading and bubbles. Economic Theory Center Research 050, Princeton University, 2013.

[70] A.-S. Sznitman. Topics in propagation of chaos. In Ecole d'été de probabilités de SaintFlour XIX - 1989, pages 165-251. Springer, 1991.

[71] J. Tirole. On the possibility of speculation under rational expectations. Econometrica, 53(6):1163-1182, 1982.

[72] W. Xiong. Bubbles, crises, and heterogeneous beliefs. Technical report, Princeton University, Princeton, NJ, 2012.

[73] S. Zhuk. Speculative bubbles, information flow and real investment. Information Flow and Real Investment, 2013. 